\title{
Hybrid Reynolds-Averaged/Large-Eddy Simulations of a Co-Axial Supersonic Free-Jet Experiment
}

\author{
R. A. Baurle* \\ NASA Langley Research Center, Hampton, Va \\ J. R. Edwards ${ }^{\dagger}$ \\ North Carolina State University, Raleigh, NC
}

\begin{abstract}
Reynolds-averaged and hybrid Reynolds-averaged/large-eddy simulations have been applied to a supersonic coaxial jet flow experiment. The experiment utilized either helium or argon as the inner jet nozzle fluid, and the outer jet nozzle fluid consisted of laboratory air. The inner and outer nozzles were designed and operated to produce nearly pressure-matched Mach 1.8 flow conditions at the jet exit. The purpose of the computational effort was to assess the state-of-the-art for each modeling approach, and to use the hybrid Reynolds-averaged/large-eddy simulations to gather insight into the deficiencies of the Reynolds-averaged closure models. The Reynolds-averaged simulations displayed a strong sensitivity to choice of turbulent Schmidt number. The baseline value chosen for this parameter resulted in an over-prediction of the mixing layer spreading rate for the helium case, but the opposite trend was noted when argon was used as the injectant. A larger turbulent Schmidt number greatly improved the comparison of the results with measurements for the helium simulations, but variations in the Schmidt number did not improve the argon comparisons. The hybrid simulation results showed the same trends as the baseline Reynolds-averaged predictions. The primary reason conjectured for the discrepancy between the hybrid simulation results and the measurements centered around issues related to the transition from a Reynolds-averaged state to one with resolved turbulent content. Improvements to the inflow conditions are suggested as a remedy to this dilemma. Comparisons between resolved second-order turbulence statistics and their modeled Reynolds-averaged counterparts were also performed.
\end{abstract}

\section{Nomenclature}

speed of sound

dissipation constant for turbulence kinetic energy

Smagorinsky constant for turbulent viscosity

constant for turbulent viscosity

distance to nearest solid surface

center jet diameter

hybrid blending function

static enthalpy

turbulence kinetic energy

turbulence length scale (Taylor microscale)

pressure

production term for turbulence kinetic energy

turbulent Prandtl number

turbulent Schmidt number

temperature

*Aerospace Engineer, Hypersonic Airbreathing Propulsion Branch, Associate Fellow AIAA.

${ }^{\dagger}$ Professor, Mechanical \& Aerospace Engineering Department, Associate Fellow AIAA. 


$\begin{array}{ll}u, v, w & \text { Cartesian velocity components } \\ x, y, z & \text { Cartesian coordinates } \\ x, r, \theta & \text { cylindrical coordinates } \\ y^{+} & \text {non-dimensional "law of the wall" coordinate } \\ Y_{m} & \text { mass fraction of species } m \\ \alpha & \text { blending function constant } \\ \delta_{i j} & \text { Kronecker delta } \\ \triangle & \text { sub grid scale filter width } \\ \eta & \text { blending function length scale ratio } \\ \kappa & \text { von Karman constant or MUSCL parameter } \\ \mu & \text { molecular viscosity } \\ \mu_{t} & \text { turbulent viscosity } \\ \nu & \text { kinematic viscosity } \\ \rho & \text { density } \\ \omega & \text { specific turbulence dissipation rate }\end{array}$

\section{Subscripts}

$i, j, k$

inj

air

computational coordinate indices

center jet injectant index

coflow air index

\section{Introduction}

Reynolds-averaged Computational Fluid Dynamics (CFD) models have become an integral part of the design and analysis of high-speed air-breathing engines. The maturation of multi-purpose CFD codes coupled with advancements in computer architectures have substantially reduced the turn-around time required to perform steady-state Reynolds-Averaged Simulations (RAS). Unfortunately, the turbulence models required to close the Reynolds-averaged equation set have not kept pace with these advancements. As a result, RAS approaches often require calibrations for key model parameters. One example is given in Ref. 1, where variations of the turbulent Prandtl and Schmidt numbers for a scramjet combustor simulation were shown to produce outcomes that ranged from engine unstart to complete flame blow-out. Similar examples that illustrate solution sensitivities to unknown RAS modeling parameters can be found in Refs. 2 and 3.

Large Eddy Simulation (LES) methods have the potential to reduce the modeling sensitivity inherent to RAS approaches, since the intent of LES is to resolve the large scale turbulent structures while modeling only the small scales. Regrettably, the computational expense of this approach (particularly when applied to configurations of interest to the high-speed propulsion community) is well beyond what can be deemed as practical by today's standards. Hybrid RAS/LES approaches offer some relief to the computational costs associated with LES. These methodologies allow LES content to be resolved in areas that require a more rigorous modeling approach, while maintaining a more cost effective RAS approach for benign regions of the flow (e.g. attached boundary layers). Hybrid approaches first appeared in the literature a decade ago, ${ }^{4}, 5$ and while many advancements have been made since then, the modeling is far from mature as evident by the vast array of hybrid approaches that have appeared in recent years. ${ }^{6,7,8,9}$ The computational expense required for a hybrid simulation, while less than that of a full LES, is still formidable when compared with steady-state RAS.

The present effort utilizes a hybrid RAS/LES approach to model a series of supersonic coaxial jet experiments 10, 11 that have been studied at the NASA Langley Research Center. These experiments involve the coaxial injection of either helium or argon into air, and CFD data (based on RAS approaches) were also obtained and compared to measurements in the works referenced above. The goals of this computational effort are to:

- Assess the capabilities of RAS and hybrid RAS/LES for predicting high speed mixing layers

- Determine the model sensitivities for both RAS and hybrid RAS/LES approaches

- Attempt to use the hybrid RAS/LES results to assess the appropriateness of models used by RAS

Measured values of composition, Pitot pressure, velocity, and rms of the velocity fluctuation are available for comparison with the simulations. Additional higher-order correlations (that are difficult to obtain exper- 
imentally) are extracted from the hybrid RAS/LES results and compared with the RAS predictions. The correlations considered include the Reynolds stress tensor and the Reynolds mass flux vector.

\section{Geometry Description and Flow Conditions}

A schematic of the coaxial nozzle assembly is shown in Fig. 1. The injectant supplied to the center jet nozzle was either a mixture of $95 \%$ helium and $5 \%$ oxygen (by volume), or pure argon. In the former case, a trace amount of oxygen was added to allow for the measurement of the streamwise component of velocity using the RELIEF ${ }^{12}$ oxygen flow-tagging technique. The internal diameter of the center jet nozzle is $10 \mathrm{~mm}$ at the nozzle exit. The center-body that forms the internal nozzle is $0.25 \mathrm{~mm}$ thick at the nozzle exit, providing a small blunt base to anchor the shear layer formed between the two nozzle streams. The coflow air nozzle has an internal diameter of $60.47 \mathrm{~mm}$, and the outer surface of this nozzle extends $12.66 \mathrm{~mm}$ farther downstream than the center-body. The $38.6^{\circ}$ juncture between the internal surface of the coflow nozzle and the conical exterior surface is sharp. Hence, there is no appreciable base region to segregate the outer jet flow from the surrounding ambient air. Further details concerning the geometry of the rig, and the methodology used for its design, can be found in Ref. 10.

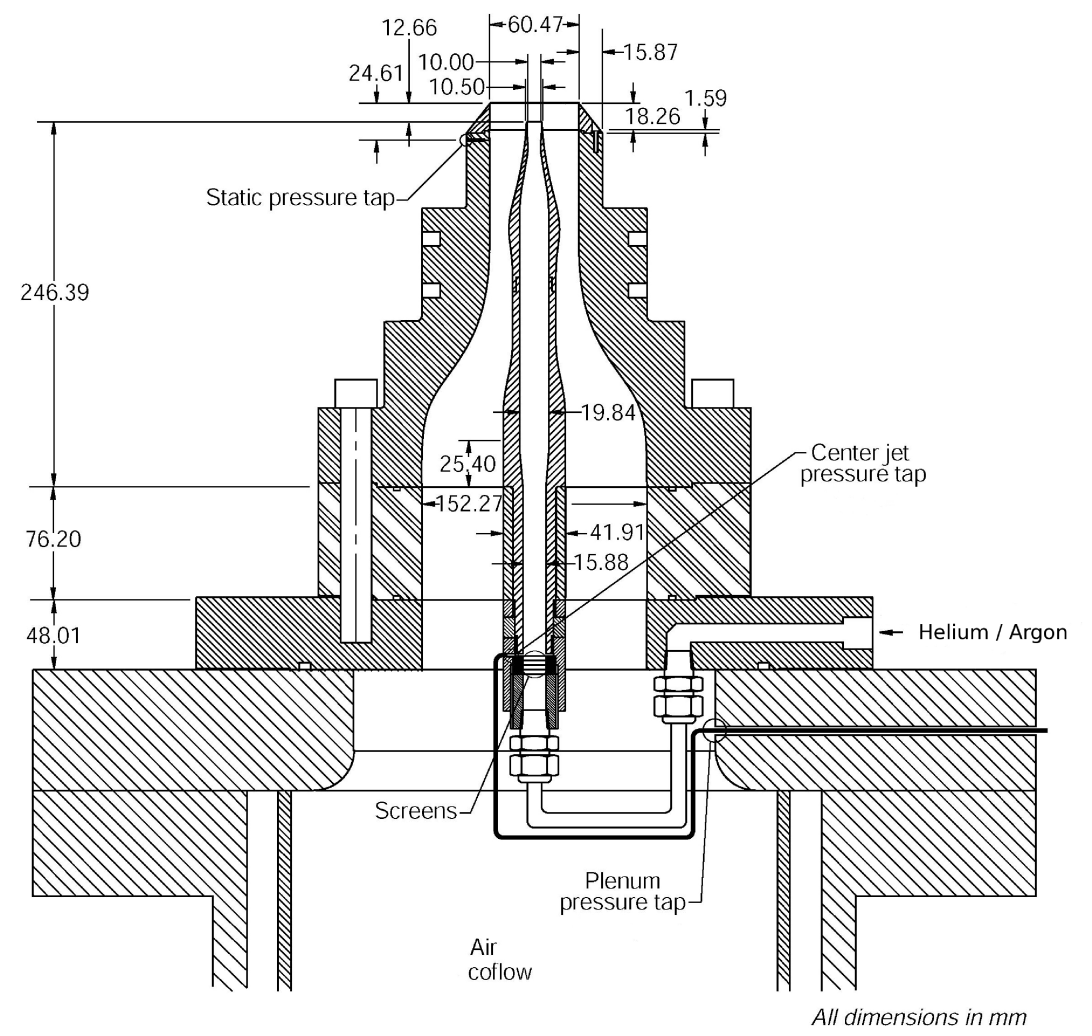

Figure 1: Schematic of the coaxial nozzle assembly

The nominal flow conditions for each experiment are given in Tables 1 and 2 along with the cited uncertainties. ${ }^{10,11}$ The thermocouples used to measure the jet temperatures were located in the gas supply lines, and the pressure taps used to measure the operating pressures were positioned as shown in Fig. 1. Although each jet has a design Mach number of 1.8, the flow velocities are markedly different due to the difference in the molecular weights for each center jet injectant. The velocity of the center jet is more than twice that of the coflow jet for the helium case (Case 1), but it is $16 \%$ lower than that of the coflow jet for the argon case (Case 2). An estimation of the convective Mach number:

$$
\frac{u_{a i r}-u_{i n j}}{a_{a i r}+a_{i n j}}
$$

yields a value of 0.7 for Case 1 and 0.16 for Case 2, respectively. Hence, one expects compressibility effects to be evident in Case 1, while Case 2 is expected to behave more like an incompressible shear layer. 
Table 1: Case 1 - Helium-Air Test Conditions

\begin{tabular}{||c|c|c|c||}
\hline \hline Nominal Conditions & Center Jet & Coflow Jet & Ambient \\
\hline \hline Mach Number & $1.8^{a}$ & $1.8^{a}$ & $0.025^{b}$ \\
\hline Total Temperature $[\mathrm{K}]$ & $305.0( \pm 15)$ & $300.0( \pm 6)$ & $294.6( \pm 6)$ \\
\hline Total Pressure $[\mathrm{kPa}]$ & $614.93( \pm 6)$ & $579.80( \pm 4)$ & $101.325( \pm 1)$ \\
\hline \hline
\end{tabular}

${ }^{a}$ nozzle design Mach number, ${ }^{b}$ value assumed for the entrained ambient flow

Table 2: Case 2 - Argon-Air Test Conditions

\begin{tabular}{||c|c|c|c||}
\hline \hline Nominal Conditions & Center Jet & Coflow Jet & Ambient \\
\hline \hline Mach Number & $1.8^{a}$ & $1.8^{a}$ & $0.025^{b}$ \\
\hline Total Temperature $[\mathrm{K}]$ & $297.9( \pm 3.5)$ & $294.3( \pm 3.5)$ & $294.6( \pm 3.5)$ \\
\hline Total Pressure $[\mathrm{kPa}]$ & $615.86( \pm 5.5)$ & $580.68( \pm 4.4)$ & $101.325( \pm 0.6)$ \\
\hline \hline
\end{tabular}

${ }^{a}$ nozzle design Mach number, ${ }^{b}$ value assumed for the entrained ambient flow

\section{Computational Methodology}

All computational results were obtained using the VULCAN (Viscous Upwind aLgorithm for Complex flow ANalysis) software package. The code solves the unsteady, conservation equations appropriate for calorically or thermally perfect gases with a cell-centered finite volume scheme. Efficient utilization of parallel architectures is realized through calls to MPI (Message Passing Interface) routines using an SPMD (Single Program Multiple Data) paradigm; a natural choice for multi-block flow solvers. Arbitrary blockto-block (non-aligned) connectivity is also available, allowing the flexibility to add or remove grid points at zonal interfaces. A variety of upwind formulations are available for evaluating the inviscid fluxes, while central differences are used for the viscous fluxes. Diagonalized Approximate Factorization (DAF) or planar relaxation (ILU) are the primary options available for steady-state simulations, while an explicit multi-stage Runge-Kutta or an implicit dual time-stepping strategy (utilizing DAF or ILU) are the options available for unsteady applications. A variety of one-equation and two-equation turbulence models exist for Reynoldsaveraged simulations. LES and hybrid RAS/LES models are also available for turbulent flows that require a more rigorous modeling effort. Further details describing the code are found elsewhere. ${ }^{13,} 14$

\section{Physical and Numerical Model Description}

Both RAS and hybrid RAS/LES computational approaches were considered in this effort. The RAS results utilized the Low-Diffusion Flux Split Scheme (LDFSS) of Edwards ${ }^{15}$ to evaluate the inviscid fluxes. The Monotone Upstream-centered Scheme for Conservation Laws (MUSCL) extrapolation parameter $(\kappa)$ was chosen as $1 / 3$ to minimize spatial truncation error, and the van Leer flux limiter ${ }^{16}$ was employed to enforce the Total Variation Diminishing (TVD) property. All of the steady-state RAS solutions were advanced in time with the diagonalized approximate factorization scheme. The turbulence model chosen was the Wilcox (1998) $k$ - $\omega$ model, ${ }^{17}$ and the wall function procedure of Wilcox ${ }^{18}$ was used to relax the grid spacing requirements near solid surfaces. A dilatation-dissipation modification ${ }^{17}$ to the turbulence kinetic energy equation was also enabled to reduce the shear layer spreading rate that has been observed at high compressible Mach number conditions. The baseline values for the turbulent Prandtl $\left(\operatorname{Pr}_{t}\right)$ and Schmidt $\left(S c_{t}\right)$ numbers, which control the turbulent transport of energy and mass, were chosen as 0.9 and 0.5 , respectively. The turbulent Schmidt number was one of the properties that was varied in parametric studies to evaluate the sensitivity of the RAS solutions to the value assumed for this coefficient.

The hybrid RAS/LES results required a somewhat different algorithmic approach to encourage the development of resolved turbulent content. In particular, the standard Total Variation Diminishing (TVD) limiters that are typically employed for RAS are overly dissipative when used for LES. This class of limiter tends to locally reduce the accuracy of the inviscid flux scheme to first-order whenever extrema of any magnitude are encountered. Essentially Non-Oscillatory (ENO) limiters offer a simple means of maintaining second-order accuracy near local extrema. This is accomplished by utilizing data at additional grid nodes to alter the stencil used to construct the inviscid flux terms. The ENO limiter used in this effort is the SONIC- 
A limiter of Suresh and Huynh, ${ }^{19}$ which can be considered as a second-order extension of the van Leer TVD limiter. All of the time-accurate hybrid RAS/LES solutions were advanced in time using a dual timestepping approach that combined the DAF method for integration in pseudo-time, with a 3-point backwards finite difference approximation for integration in real-time. The values selected for the physical time-step and sub-iteration CFL constraint were $0.05 \mu s$ and 50.0, respectively. The time-step was chosen based on a cell residence time constraint to ensure that turbulent structures would not traverse more than one grid cell per iteration. The sub-iteration process was considered converged when the residual error dropped at least 2.5 orders of magnitude. This level of convergence typically required 4-7 sub-iterations for each physical time-step.

The hybrid RAS/LES methodology used in this effort builds on the previous work discussed in Ref. 6 . This framework was designed to enforce a RAS behavior near solid surfaces, and to switch to an LES behavior in the outer portion of the boundary layer and free shear regions. Hence, this formulation can be thought of as a wall-modeled LES approach, where RAS is used as the near-wall model. The basic idea is to blend any trusted RAS eddy viscosity with a desired LES Sub-Grid Scale (SGS) viscosity, along with any transport equations that involve a common RAS and SGS property. In this effort, the Wilcox (1998) $k-\omega$ RAS model ${ }^{17}$ was blended with the one-equation model of Yoshizawa. ${ }^{20}$ The Yoshizawa model involves an evolution equation for the SGS turbulence kinetic energy, hence the blended expressions that are appropriate for this model combination are:

$$
\begin{aligned}
\text { Hybrid RAS/SGS viscosity } & =(F)[\text { RAS viscosity }]+(1-F)[\text { SGS viscosity }] \\
\text { Hybrid RAS/SGS k-equation } & =(F)[\text { RAS k-equation }]+(1-F)[\text { SGS k-equation }]
\end{aligned}
$$

where $F$ is a blending function that varies between 0 and 1 . Note that the transport equation for the RAS specific dissipation rate $(\omega)$ does not have an SGS counterpart. Hence, the blending is not applied to this equation, and all of the terms in this equation that involve the eddy viscosity are evaluated based on RAS relationships.

The motivation behind the development of this hybrid RAS/LES framework is two-fold. First, the blending of two independent RAS and LES closure models offers the flexibility of having an optimized set of closure equations for both RAS and LES modes. The second (and more critical) driving factor was the desire to alleviate the difficulties associated with the design of grid topologies that are appropriate for purely griddependent blending paradigms such as those used for Detached Eddy Simulation (DES). ${ }^{4}{ }^{21}$ The movement away from simple grid-dependent blending strategies has gained momentum in recent years. ${ }^{7,8}$ In fact, even the developers of DES are now promoting an "improved" version of their scheme termed Delayed Detached Eddy Simulation (DDES) ${ }^{8}$ that involves flow-dependent functions to switch between RAS and LES regimes.

The blending function $(F)$ used in this effort is based on the ratio of the wall distance $d$ to a modeled form of the Taylor microscale $(\ell)$ :

$$
\begin{aligned}
F & =\frac{1}{2}\left\{1-\tanh \left[5\left(\frac{\kappa}{\sqrt{C_{\mu}}} \eta^{2}-1\right)-\phi\right]\right\} \\
\ell & =\sqrt{\frac{\nu}{C_{\mu} \omega}} \\
\eta & =\frac{d}{\alpha \ell}
\end{aligned}
$$

where $\kappa$ is the von Karman constant $(0.41), C_{\mu}$ is $0.09, \alpha$ is a user-defined model constant, and $\phi$ is set to $\tan ^{-1}(0.98)$ to force the balancing position of $F$ (i.e. the position where $\left.\kappa \eta^{2}=\sqrt{C_{\mu}}\right)$ to 0.99 . The value chosen for $\alpha$ provides control over the $y^{+}$position where the average LES to RAS transition point (defined as $F=0.99$ ) occurs. If resolved LES content is desired for an attached boundary layer, then this constant should be set such that the transition point occurs in the region where the boundary layer wake law starts to deviate from the log law. If the transition point is enforced at a lower $y^{+}$value $(e . g$. well within the $\log$ law region), a dual log layer can appear. ${ }^{22,23}$ Conversely, if the transition point is enforced at a $y^{+}$value that extends well into the defect layer, then the level of resolved turbulence will be limited. Details on a procedure to analytically determine the value for $\alpha$ that corresponds to a target $y^{+}$value is described in Ref. 23. 


\section{Grid Details}

A two-dimensional (axisymmetric) grid was generated for the steady-state Reynolds-averaged solutions (see Fig. 2). This grid consisted of just under 250,000 cells divided across five structured grid zones. The computational domain extended 150 center jet diameters $(D)$ downstream of the jet exit, and between 40 and 70 jet diameters in the radial direction. The internal flowfield within the concentric nozzles was also included as part of the computational domain to allow for the development of boundary layers. The last experimental data stations were located 26.101 (Case 1) and 45.276 (Case 2) jet diameters downstream of the center jet exit plane. Hence, most of the grid was clustered between the jet exit and an $x / D$ of 50 . The portion of the computational domain downstream of $x / D=50$ was added to provide plenty of space for the jet flow to reach a subsonic state, which ensured that the specified pressure outflow boundary condition remained well-posed. The far-field boundary was also placed many tens of jet diameters away from the domain of interest to minimize any chance of data corruption that might occur via wave reflections off of the characteristic inflow condition that was applied along this boundary. The nozzle stagnation conditions given in Tables 1 and 2 were applied at each nozzle inflow plane. The operating total temperature of each nozzle was nominally the same as the room temperature, so all solid surfaces were assumed to be adiabatic. The grid was clustered to all solid surfaces at a level appropriate for the use of wall functions $\left(y^{+} \leq 36\right)$. Finally, the entire grid system was broken up into a total of 179 grid blocks which yielded excellent load balance statistics for up to 64 processors.

An azimuthal slice of the three-dimensional grid generated for the hybrid RAS/LES cases is shown in Fig. 3. The boundary conditions and the extent of the computational domain was identical to that used for the axisymmetric RAS cases. The gridding strategy, however, was altered to reflect the change in computational algorithm. LES requires the use of grid cells that are roughly isotropic to allow for the development and sustainment of resolved turbulent structures. Hence, the streamwise grid spacing had to be reduced from that utilized for the pure RAS. This requirement, coupled with the fact that the full 3-D flowfield must be solved, forced a concession to be made to keep the computational costs within reason. The compromise that was accepted was to provide a level of grid resolution capable of supporting LES only up to an $x / D$ of 25 (highlighted in red). This domain captures all but the last experimental station for Case 1, and 13 of the 16 stations for Case 2. Non-aligned zonal interfaces (patches) were inserted at $x / D$ stations of 25,50 , and 100 to systematically remove grid nodes in the radial direction. A patch interface was also inserted in the ambient air region to coarsen the streamwise spacing in the far-field where axial refinement is not required. Each patch interface is denoted by a solid black line in Fig. 3. In addition to substantially reducing the computational costs, the coarsening process performed at large $x / D$ tends to dissipate the large scale vortical structures that propagate out of the LES domain of interest. This reduces the likelihood of an ill-posed boundary condition appearing at the outflow plane. An H-O mesh topology was chosen for the cross-flow planes to avoid the numerical difficulties associated with collapsed faces that would result from a classic polar topology. This feature of the grid is illustrated in the three-dimensional view of the LES domain shown in Fig. 4. Finally, the entire grid system was broken up into a total of 1669 grid blocks which resulted in excellent load balance statistics for up to 360 processors.

\section{Reynolds-Averaged Results}

Contours of helium mass fraction and Mach number extracted from the baseline RAS $\left(S c_{t}=0.5\right)$ are shown in Fig. 5. The helium mass fractions have been normalized by the the center jet value (95\% by volume corresponds to $70.39 \%$ by mass):

$$
Y_{H e} \Rightarrow Y_{H e} / 0.7039
$$

for all of the results that follow to simplify the analysis and comparisons with the argon cases. The helium contours show that the potential core of the center jet persists for approximately 12 jet diameters, and the center-line helium mass fraction in the plume that forms downstream of this station decays to a value of 0.115 at an $x / D=50$. The Mach contours show that both nozzle streams are nearly pressure matched. Although not evident at this scale, a pair of counter-rotating separation bubbles are present behind the small blunt base of the center-body. The weak expansion fan that is seen on either side of the base region forms as the jets negotiate around this separated flow zone. A weak recompression shock then develops as the flow is forced to turn back on itself at the close-off point of the recirculation bubble. The conical shock that forms in the center jet flowfield steepens as it approaches the axis of symmetry, resulting in a Mach disk at the axis. As will be shown later, the total pressure loss that occurs across this shock wave is also evident in the measured Pitot pressure surveys. 


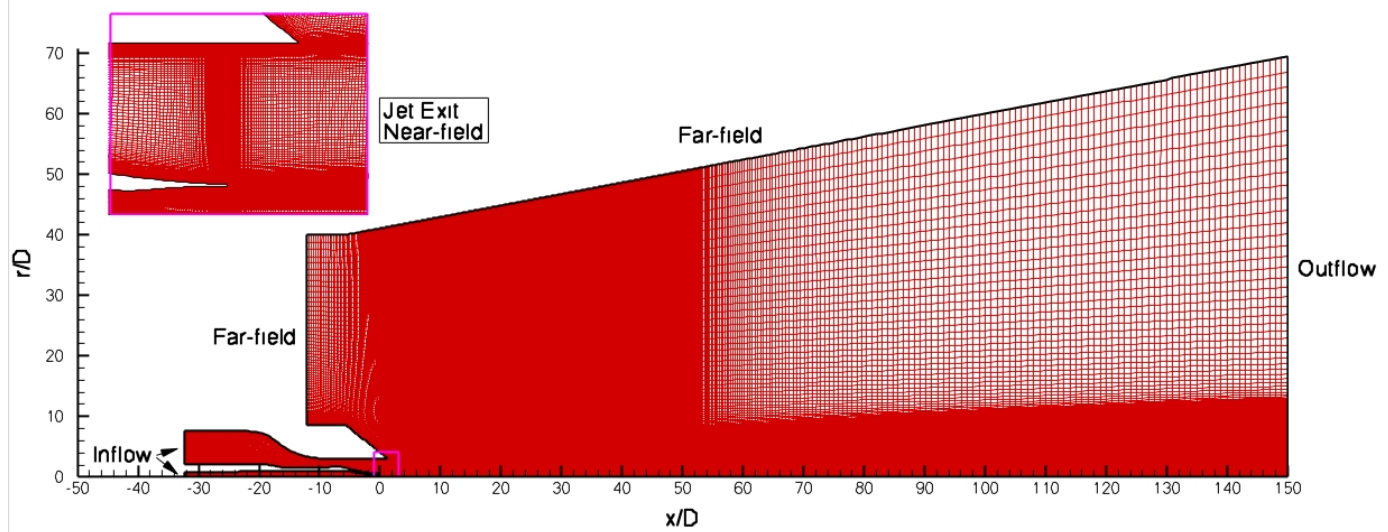

Figure 2: Axisymmetric grid utilized for RAS (247368 cells)

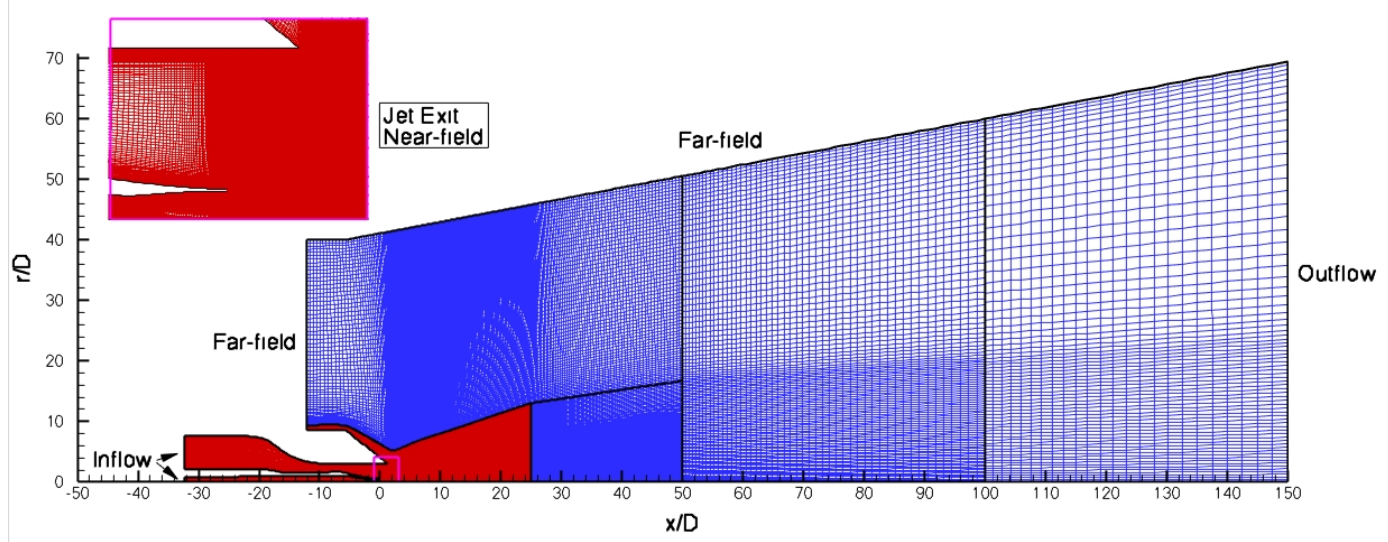

Figure 3: Azimuthal slice of the three-dimensional grid utilized for hybrid RAS/LES (43,285,632 cells)

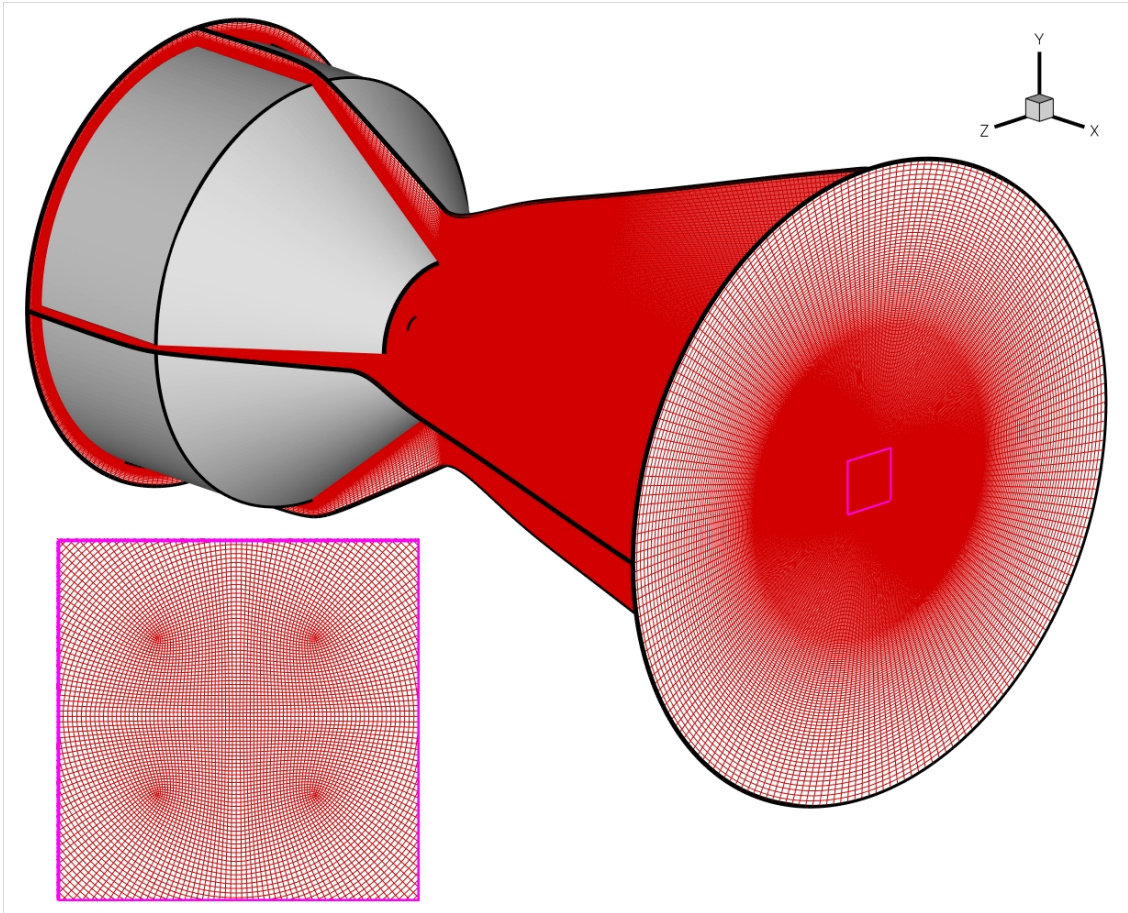

Figure 4: Isometric visualization of the LES-resolved portion of the hybrid RAS/LES grid 


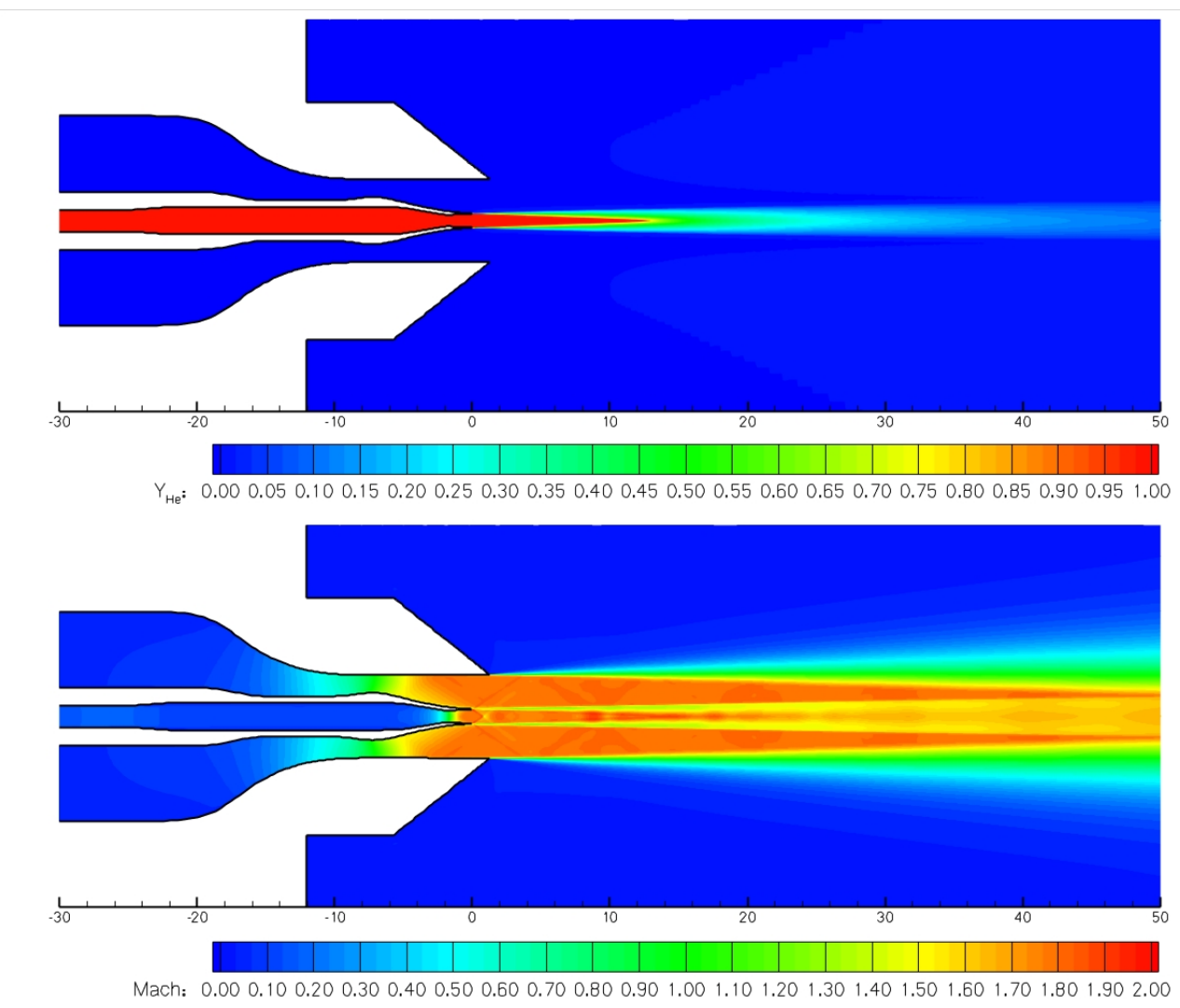

Figure 5: Contours of normalized helium mass fraction and Mach number for the baseline RAS

Measured helium mass fraction profiles are compared with the RAS results in Fig. 6. The measurements of injectant composition were made using a gas sampling probe as detailed in Ref. 10. In general, the baseline simulation over-predicted the rate of mixing between the coaxial streams. Hence, an additional simulation was performed with the $S c_{t}$ value increased by a factor of 2 (to 0.25 ). The third result shown in this figure is a repeat of the baseline condition, but simulated on a coarse grid (factor of 2 coarsening in each coordinate direction). Comparisons are made at 8 axial stations downstream of the center jet exit plane. The results extracted from the baseline $\left(S c_{t}=0.5\right)$ simulation over-predicted the measured growth rate of the mixing layer for all but the first experimental station. It should be noted that the RAS model used in this effort included a dilatation-dissipation modification ${ }^{17}$ to reduce the shear layer spreading rate for compressible mixing layers. This leaves the turbulent Schmidt number (which controls the rate of turbulent mass diffusion) as the remaining parameter that can be adjusted to reduce the mixing rate. The results obtained when doubling the $S c_{t}$ value (which reduces the modeled turbulent diffusion term by a factor of 2) agree well with the measurements. In fact, the results obtained with $S c_{t}=1.0$ matched nearly every measured result to within the precision of the measurement device of $\pm 1.0-1.5 \%$. The effect of coarsening the grid resolution had a much smaller impact on the predicted results, indicating that the level of grid convergence is well within the uncertainty bounds of the physical models employed.

Measured Pitot pressure profiles ${ }^{10}$ are compared with the RAS results in Fig. 7. The large deficit in Pitot pressure near $r / D=0.5$ is a result of viscous losses from the upstream boundary layers. The small deficit seen near the axis of symmetry at the first four axial stations is a result of the Mach disk formed by the coalescence of the conical shock front that was discussed previously (see Fig. 5). The measured value of this deficit is slightly larger than the predicted value. The Pitot pressure deficit is also under-predicted for the coflow shock structure that appears near $r / D=1.1$ at the first axial station. In general, the best agreement with measurements is given by the $S c_{t}=1.0$ data. The level of agreement seen between this simulation and the measured values is within the measurement uncertainty (based solely on the transducer error) of $\pm 0.5 \%$ at nearly every measured point for $x / D<15$. Downstream of this station, the $S c_{t}=1.0$ Pitot data under-predicts the measured values near the axis, and over-predicts them near the lower edge of the shear layer present between the coflow jet and the ambient surroundings. Once again, the differences noted between the fine and coarse grid solutions is much smaller than that associated with a modification 
of the turbulent Schmidt number.

Contours of argon mass fraction and Mach number extracted from the baseline RAS $\left(S c_{t}=0.5\right)$ are shown in Fig. 8. The argon results show a much longer potential core than that for the helium case. In fact, the potential core in the simulations extends beyond the $x / D=50$ station shown in Fig. 8. The slower mixing rate between the two jet streams is a result of the reduced shear associated with Case 2 . The velocity of the argon stream is only $16 \%$ lower than the coflow stream. This is contrasted with the helium case, where the velocity of the helium stream was more than twice that of the coflow stream. The Mach contours clearly show that the nozzle streams are nearly pressure matched, and the near-field flow structures are practically identical to those discussed previously for Case 1. The far-field shock/expansion pattern is quite different, however, due to the reduced spreading rate of the mixing layer.

Measured argon mass fraction profiles ${ }^{11}$ are compared with the RAS results in Fig. 9. The baseline argon simulation under-predicted the rate of mixing between the coaxial streams. This is in contrast to the helium case where the baseline modeling parameters over-predicted the growth rate of the mixing layer. Hence, an additional simulation was performed with the turbulent Schmidt number reduced by a factor of 2. A third simulation was performed with the baseline modeling parameters using the coarse grid to evaluate the grid dependence. Comparisons are made at the same 8 axial stations used to evaluate the helium injection data. The results obtained when reducing the $S c_{t}$ value had the desired effect of reducing the length of the potential core of the argon jet. However, this adjustment also resulted in a peculiar inflection point near the outer edge of the mixing layer that is not evident in the measurements. The reduced Schmidt number results also over-predicted the spreading rate of the mixing layer for $x / D<18$. Overall, the best result was obtained with the baseline modeling parameters, which predicted the correct spreading rate of the shear layer for $x / D<18$. The convective Mach number for the mixing layer was estimated to be 0.16 , so an additional simulation (not shown) was performed with the dilatation-dissipation modification disabled to ensure that the model was not impacting the predictions. As expected, the compressibility correction had no impact on this simulation. One interesting feature noticed in the experimental data is an increased deviation from symmetry that begins to appear at the $x / D=12$ station. This happens to be the station where simulations begin to deviate from the measurements. The experimental probes were traversed over the entire coaxial flowfield (i.e. data was gathered for both positive and negative $y$-values relative to the jet axis). Since the data should be axisymmetric in the mean, the measured data was plotted against the absolute value of $y$ in this work, which effectively results in two sets of "radial" data at each experimental station. The deviation from symmetry is much larger for Case 2 than for Case 1, which might have implied a slight misalignment of the jet assembly during the argon tests. ${ }^{11}$ As was seen for the helium injection conditions, the predictions offered by both the fine and coarse meshes were quite similar.

Measured Pitot pressure profiles ${ }^{11}$ for Case 2 are compared with the RAS results in Fig. 10. The nearfield Pitot profile features are identical to those seen for Case 1, as implied by the Mach contours that were discussed earlier. In general, the level of agreement seen between the simulations and the measured values is within the measurement uncertainty for $x / D<15$. Downstream of this station, the computed Pitot data shows sharper features, which is a direct result of the under-prediction of the mixing processes at this point in the flowfield. It is interesting to note that the over-prediction of the Pitot pressure near the lower edge of the shear layer present between the coflow jet and the ambient surroundings is larger that what was seen for Case 1. The shear between the coflow jet and the ambient environment is practically identical to that for the helium condition. Hence, the under-prediction of the argon/air mixing layer has evidently modified the shock system in the outer jet enough to alter the behavior of the coflow/ambient air shear layer.

\section{Hybrid RAS/LES Results}

The coaxial flowfields examined in this effort have three distinct flow regions: center jet, coflow jet, and the ambient surroundings. The core velocity in the coflow jet was $480 \mathrm{~m} / \mathrm{s}$, and the center jet values were $1100 \mathrm{~m} / \mathrm{s}$ (helium) and $400 \mathrm{~m} / \mathrm{s}$ (argon). The ambient air region was almost stagnant. This presented a problem in that the hybrid RAS/LES approach requires a time-accurate integration with a time-step small enough to temporally resolve turbulent structures at scales representative of a computational cell width. A time-step appropriate for resolving a turbulent structure in the jet flow is given by the following estimate:

$$
\triangle t \approx \frac{\triangle x}{\max \left(u_{i n j}, u_{a i r}\right)}
$$



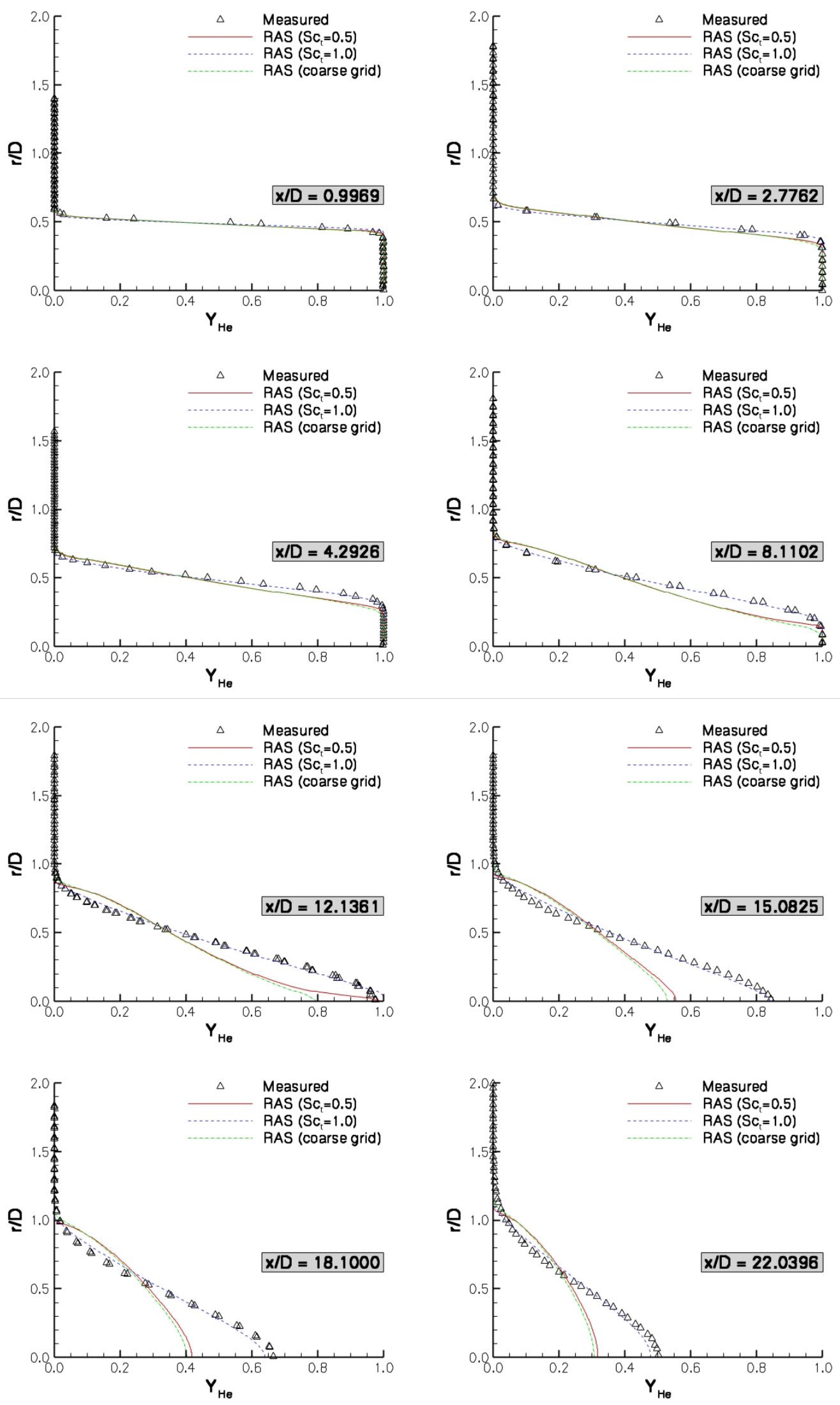

Figure 6: Comparison of normalized helium mass fraction (RAS predictions) with measured values 

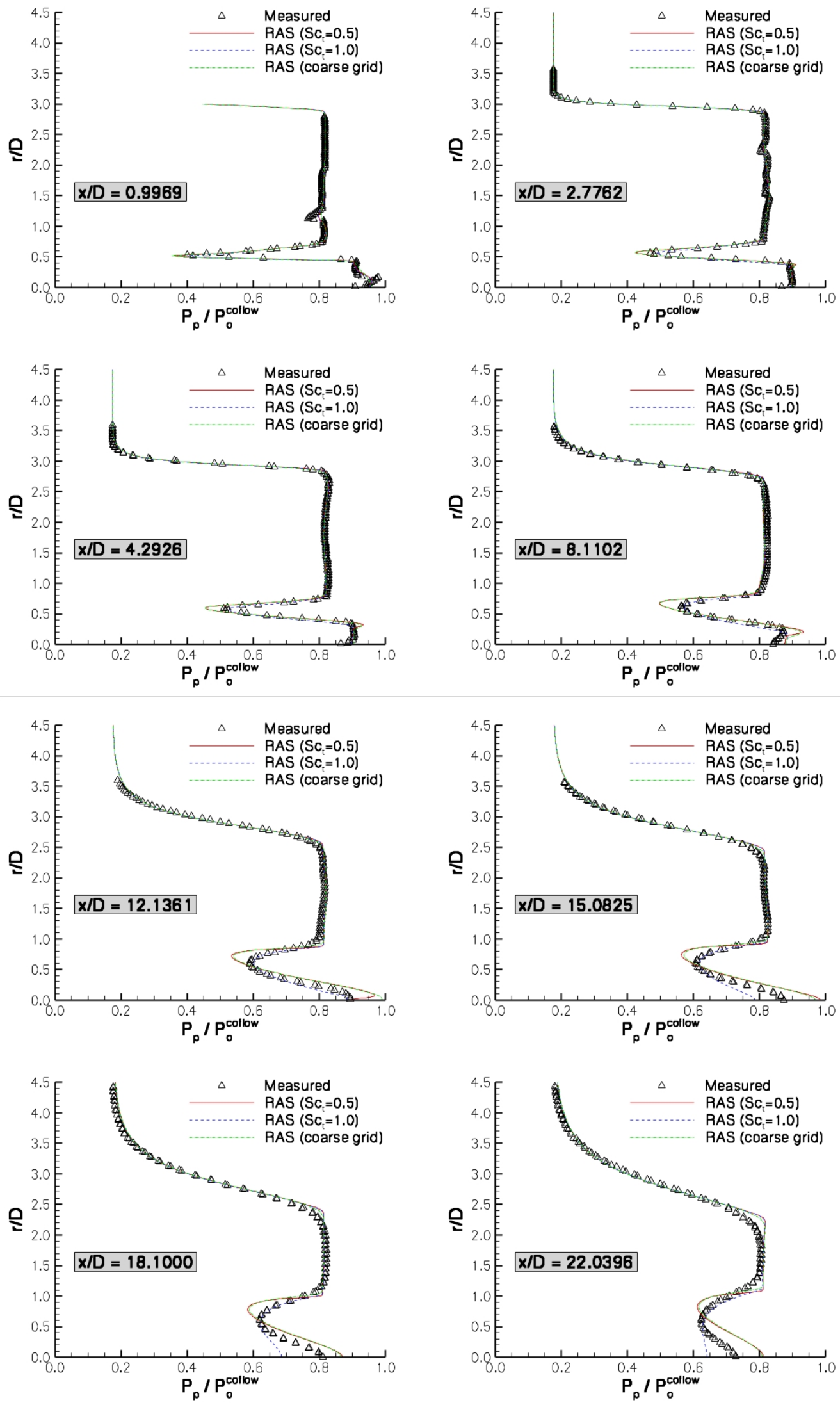

Figure 7: Comparison of Case 1 Pitot pressure (RAS predictions) with measured values 


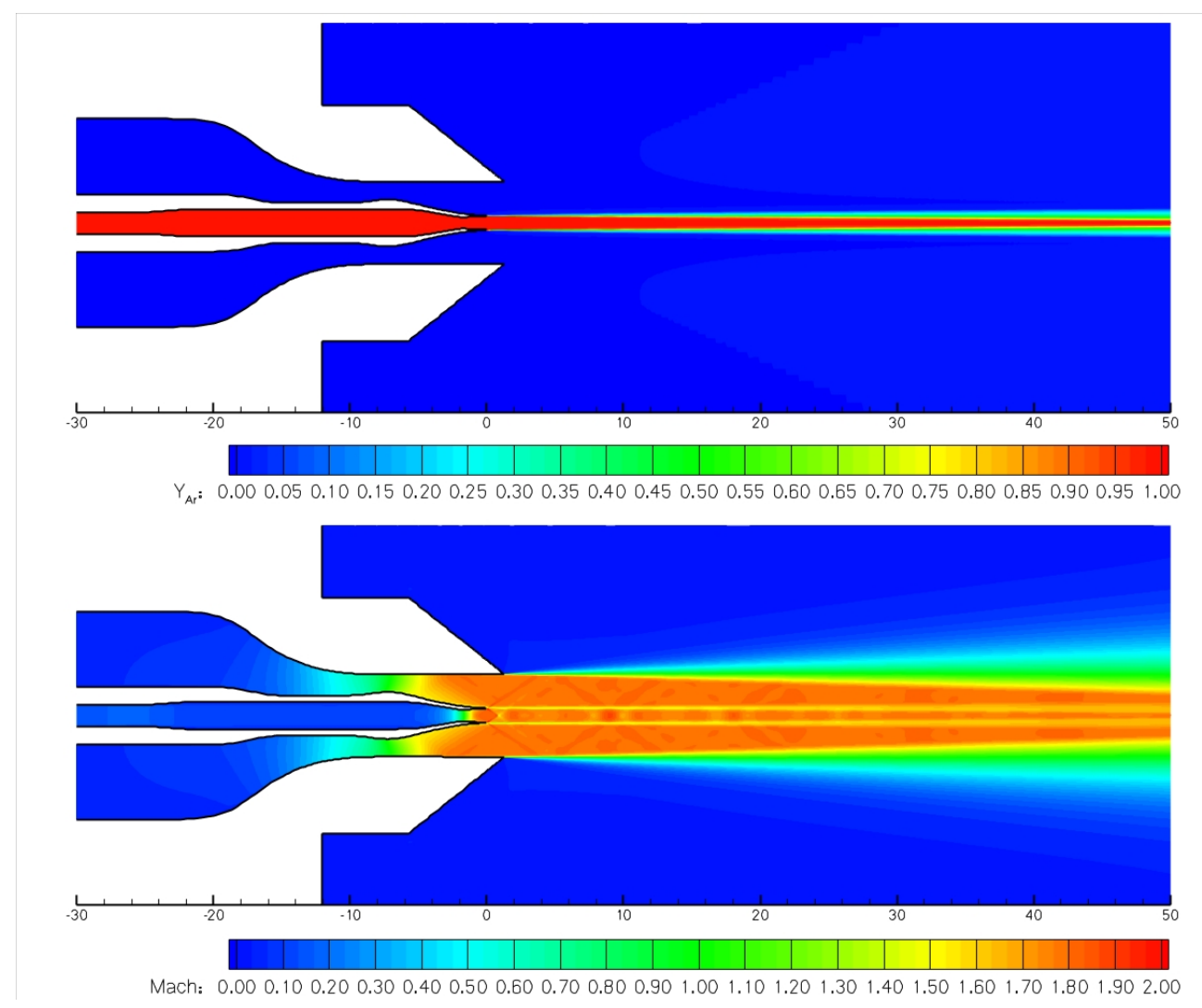

Figure 8: Contours of argon mass fraction and Mach number for the baseline RAS

Based on the disparate characteristic velocities between the jet flows and the ambient air, this time-step would require hundreds of iterations to transport a particle across one cell length in the ambient air region. Fortunately, the shear layer of interest is the mixing layer between the supersonic jets rather than the shear layer present between the coflow jet and the ambient air. Hence, the time scale dilemma was dealt with by forcing the hybrid RAS/LES model to remain in RAS mode for the outer portion of the coflow jet and ambient region. In particular, an initial solution was obtained for the entire flowfield using a steady-state RAS. The calculation was then restarted with the hybrid RAS/LES approach with the blending function forced to unity for $r / D>2$. This value was found to be large enough to contain all convecting coherent structures formed in the mixing layer between the two jet flows for the LES domain of interest.

The form of SGS closure used in this effort was based on the one-equation Yoshizawa ${ }^{20}$ model:

$$
\begin{aligned}
\frac{\partial}{\partial t}(\rho k)+\frac{\partial}{\partial x_{j}}\left(\rho k u_{j}\right) & =\frac{\partial}{\partial x_{j}}\left[\left(\mu+\mu_{t}\right) \frac{\partial k}{\partial x_{j}}\right]+P_{k}-C_{d} \rho \frac{k^{\frac{3}{2}}}{\triangle} \\
\mu_{t} & =C_{\mu} \rho k^{\frac{1}{2}} \triangle
\end{aligned}
$$

However, two noteworthy modifications to the model were made for this effort. The first modification involves the definition of the filter width, $\triangle$. Traditional LES practitioners have tended to work with truly isotropic grids, justifying the use of a simple isotropic definition for the filter width (e.g. the cubed root of the cell volume). Grids utilized for configurations of engineering interest, however, will have a some level of anisotropy. Hence, hybrid RAS/LES practitioners tend to prefer the following anisotropic definition for the filter width:

$$
\triangle=\max \left(\triangle_{i}, \triangle_{j}, \triangle_{k}\right)
$$

In this expression, $\triangle_{i}, \triangle_{j}$, and $\triangle_{k}$ denote the width along each computational direction of a threedimensional hexahedral grid cell. The second modification affects the level of SGS viscosity in LES regions. At equilibrium (defined when production balances dissipation in Eq. 6a), the Yoshizawa model reduces to an algebraic Smagorinsky closure model. The effective Smagorinsky constant $\left(C_{s}\right)$ implied by the model under 

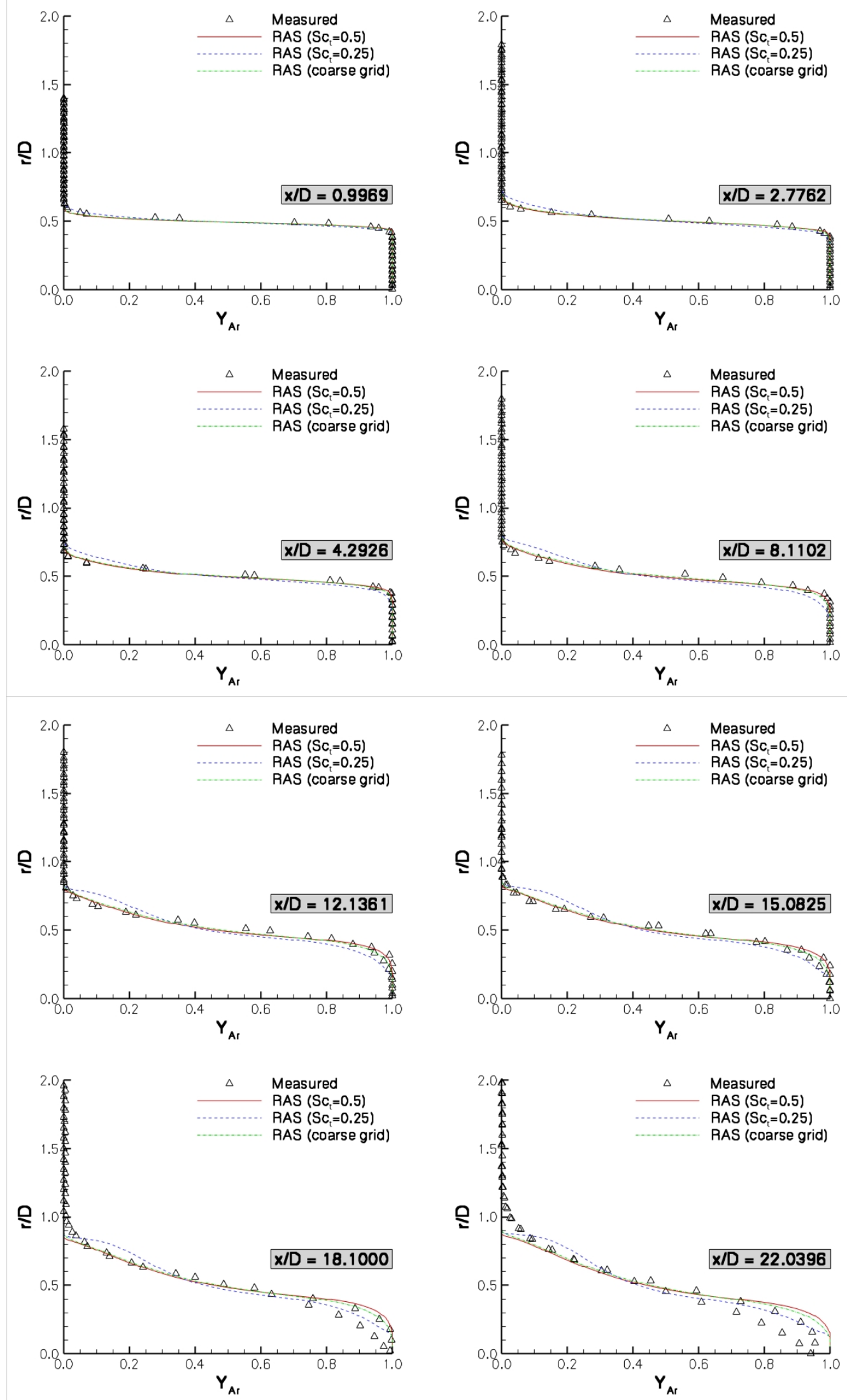

Figure 9: Comparison of argon mass fraction (RAS predictions) with measured values 

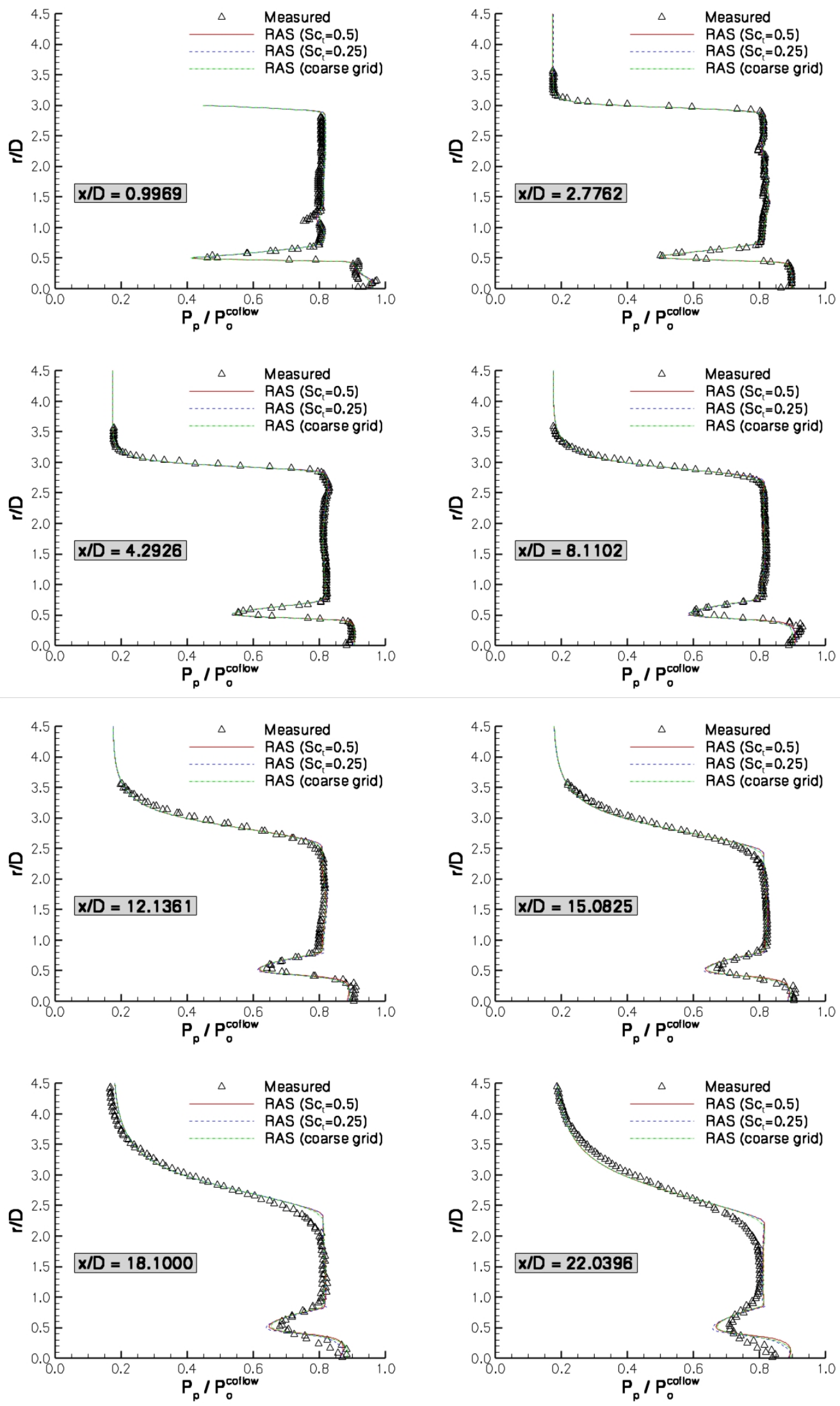

Figure 10: Comparison of Case 2 Pitot pressure (RAS predictions) with measured values 
equilibrium conditions is given by the following expression:

$$
\left(C_{s}\right)^{2}=\sqrt{2} C_{\mu}\left(\frac{C_{\mu}}{C_{d}}\right)^{\frac{1}{2}}
$$

The default values given in Ref. 20 for $C_{\mu}$ and $C_{d}$ are 0.05 and 1.0, respectively. The value of $C_{s}$ implied by these values is 0.126 . There is no universal value for this Smagorinsky constant, but the LES community has typically endorsed values on the order of 0.2 for homogeneous turbulence and 0.065 for shear flows. ${ }^{24}$ Initial calculations used a reduced value of 0.02075 for $C_{\mu}$ to recover the "accepted" Smagorinsky constant for shear flows. This value, however, proved to be too dissipative within the present second-order upwind numerical framework. The numerical dissipation is on the same order as the SGS viscosity $\left(\triangle^{2}\right)$, hence one might expect lower values to be required. A value of $C_{\mu}=0.00823$ was found to readily unlock significant levels of resolved turbulence energy in the present simulations. This value enforces a Smagorinsky constant of 0.0325 (half the recommended value for shear flows) under equilibrium conditions, and was selected as the baseline setting.

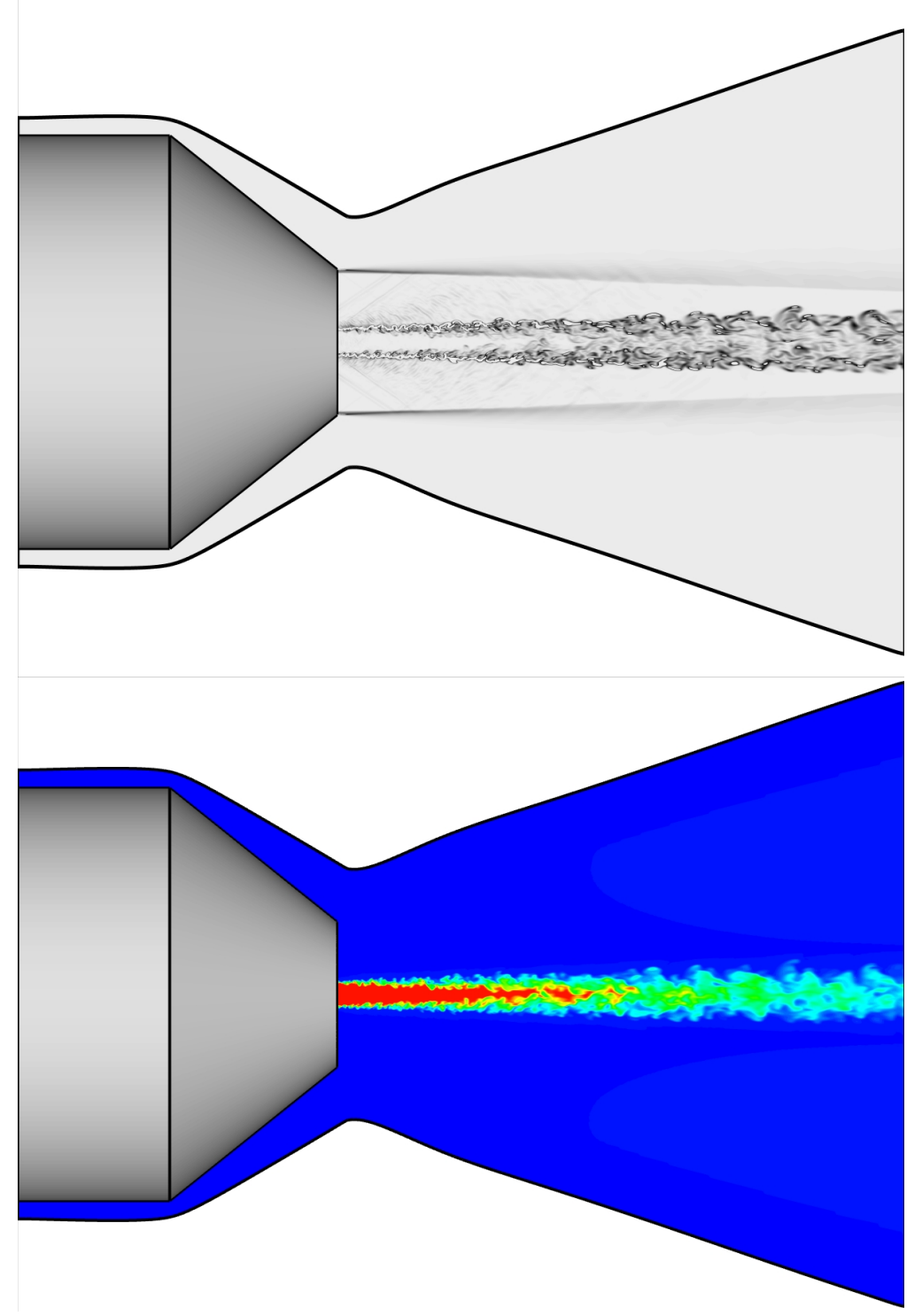

Figure 11: Instantaneous Schlieren and normalized helium mass fraction contours (hybrid RAS/LES)

An instantaneous snapshot of the flowfield for Case 1 is shown in Fig. 11. The top image displays a numerical Schlieren of the flow structure, and the bottom image shows the normalized helium mass fraction 
contours. A significant level of resolved turbulent content was captured in the simulation. The recirculation zone behind the base of the center-body provided a source of large scale unsteadiness that rapidly triggered Kelvin Helmholtz instabilities in the shear layer. These instabilities transitioned the flow from a fully modeled Reynolds-averaged state exiting the nozzle, to a primarily resolved turbulent state a few jet diameters downstream of the nozzle exit plane. Unsteady shock and expansion waves are seen reflecting through the jet structure. The shear layer between the outer jet and ambient air maintained the desired steady-state Reynolds-averaged behavior due to the forced usage of the RAS equations outside of $r / D=2.0$. The high level of shear associated with this operating condition provided a strong mechanism for turbulence production as evident by the rich array of vortical structures seen in the helium contours. These structures provide the turbulent "stirring" that leads to enhanced mixing by stretching the interface between the air and injectant.

Prior to gathering any statistics, time integrations were performed for two complete flow-through times to flush out the initial transients during the transition from the converged RAS solution to the hybrid RAS/LES result. This relatively small time interval was sufficient due to a lack of any large regions of separated flow, and the fact that the ambient region was essentially frozen in its Reynolds-averaged state. At this point, running averages were computed and stored after each iteration. In order to reduce the integration time required to gather meaningful statistics, a combination of temporal and spatial (in the circumferential direction) averaging was performed. The spatial-average was made possible by a three-step process. The first step was to interpolate the ensemble-averaged data onto a polar grid topology (in the cross-flow plane) at the experimental streamwise stations. The second step involved a coordinate transformation of the flow data to a cylindrical coordinate system $(x, y, z \rightarrow x, r, \theta)$. The final step was to average the transformed variables along the azimuthal curves of constant radius (which corresponds to the spatially homogeneous direction). All three steps were performed using the TECPLOT post-processing package ${ }^{25}$ and automated using the TECPLOT macro scripting language.

The averaged hybrid RAS/LES helium mass fraction profiles are compared with the measurements in Fig. 12. In general, the predictions show a shear layer growth rate that is more rapid than that implied by the measurements. As mentioned previously, the SGS model coefficients were adjusted to promote the onset of flow instabilities. It appears that this adjustment leads to an inappropriate level of modeled viscosity (i.e. a value that is too low) once the Kelvin Helmholtz instabilities have transitioned to a fully turbulent state. This situation might be improved if resolved turbulent structures were allowed to develop at the nozzle exit plane. This would remove the delay in transition that is associated with the conversion of modeled Reynolds-averaged turbulence to resolved LES content, and would allow a larger Smagorinsky coefficient to be utilized. One reasonably economic approach to this task is to implement recycling/rescaling techniques. ${ }^{26,27,28}$ This will be the focus of future efforts. A comparison of the averaged hybrid RAS/LES results with those obtained using pure RAS at the default $\left(S c_{t}=0.5\right)$ condition show many similarities. This particular RAS simulation also over-predicted the shear layer growth rate, but one noteworthy difference between the results is the manner in which the helium mass fraction asymptotically approaches zero at the edge of the mixing layer. RAS approaches are notorious for producing a sharp non-physical interface at the edge of the shear layer. The hybrid RAS/LES results show a much smoother asymptotic decay that is more representative of the measurements. Statistical convergence has been assessed by comparing the statistics extracted over successively larger iteration intervals. The statistics extracted over twenty and thirty thousand iterations show practically identical results. Another useful statistical convergence check is to examine the averaged azimuthal velocity component. This value should approach zero as the statistics are converged. The maximum absolute value of azimuthal velocity (averaged over 30,000 cycles) was $2.5 \mathrm{~m} / \mathrm{s}$, or roughly $0.2 \%$ of the streamwise velocity.

The averaged Pitot pressure profiles are compared with the measurements in Fig. 13. These results reinforce the assertion that the mixing layer growth rate has been over-predicted, particularly at stations downstream of an $x / D$ of 4 . Although the deficit in Pitot pressure profile is broader across the shear layer, the qualitative features of the profile shape mimics that of the measurements. This was not the case in the RAS results that over-predicted the level of mixing. The finer details of the flow structure, e.g. the slight pressure deficit due the the Mach disk at the axis, are also evident in the hybrid RAS/LES data. As was the case with the helium mass fraction statistics, the averaged Pitot pressure profiles for both averaging intervals show practically identical results, suggesting that the first-order moments are well converged.

An instantaneous snapshot of the flowfield for Case 2 is shown in Fig. 14. The top image displays a numerical Schlieren of the flow structure, while the lower image displays the argon mass fraction distribution. The Kelvin Helmholtz instabilities are clearly evident in both images and persist for many jet diameters before 

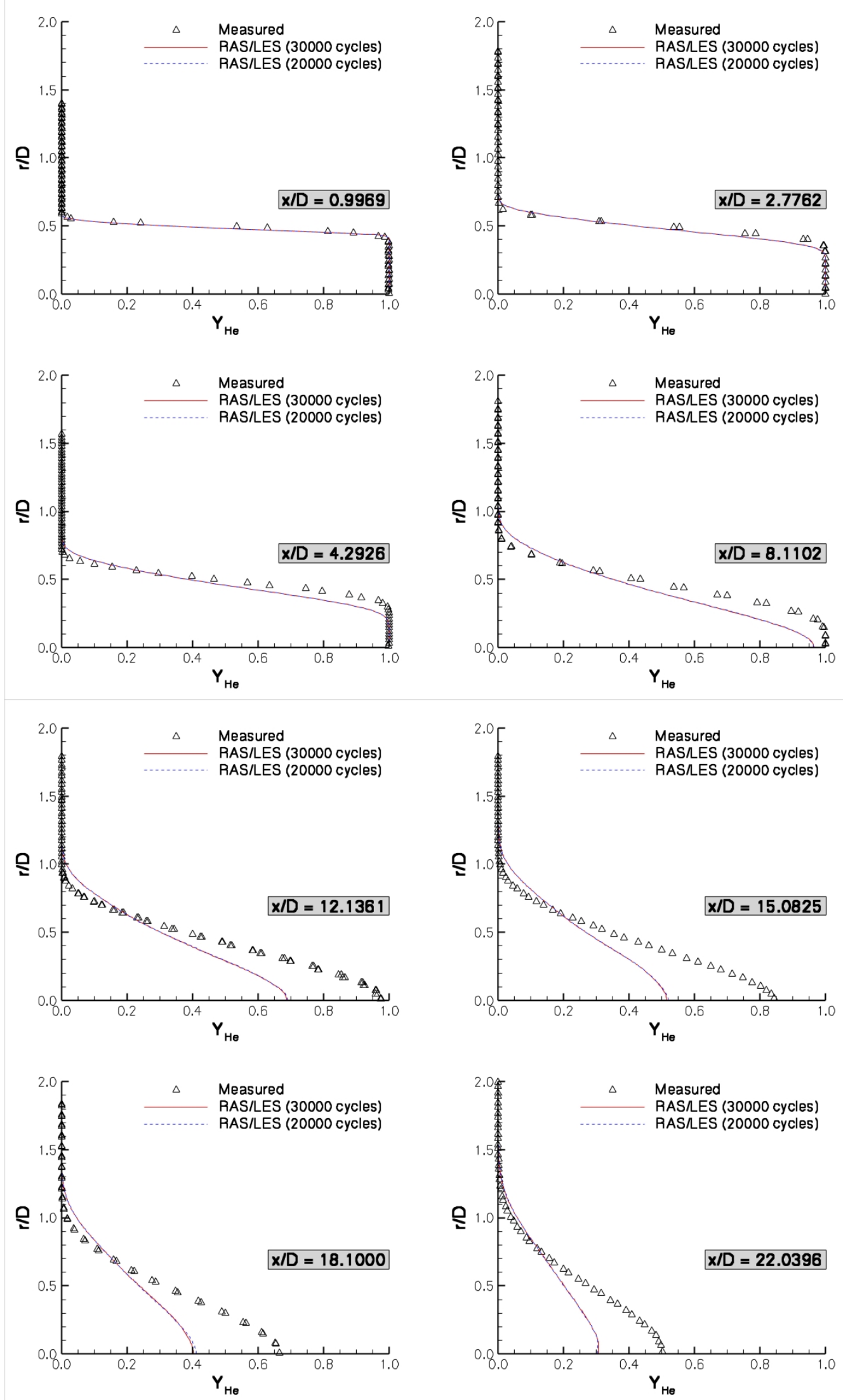

Figure 12: Comparison of normalized helium mass fraction (averaged hybrid RAS/LES predictions) with measured values 

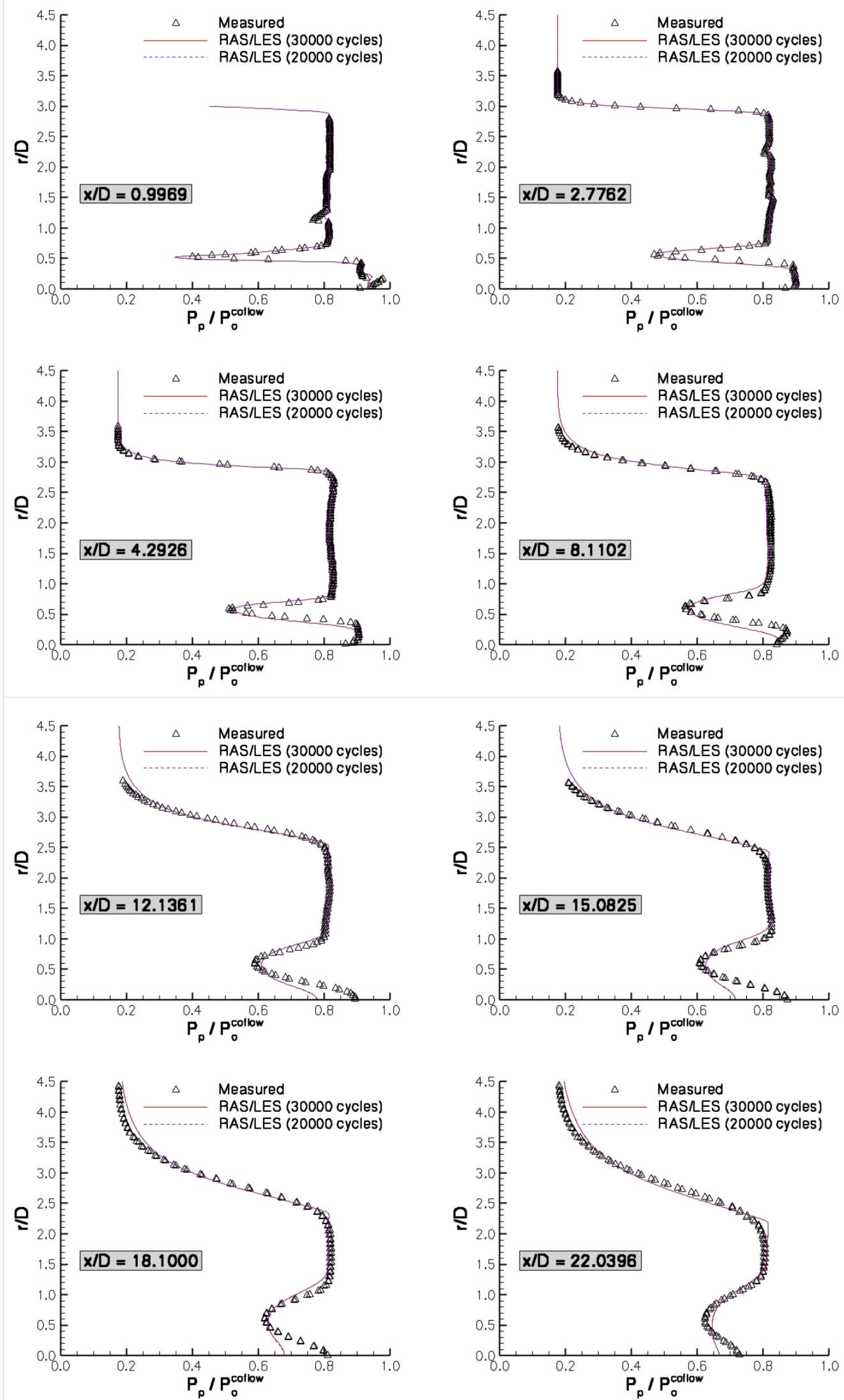

Figure 13: Comparison of Case 1 Pitot pressure (averaged hybrid RAS/LES predictions) with measured values 
breaking down into fully three-dimensional turbulent structures. This transition process occurs near an $x / D$ of 10 or 12, where the last visible wave structure appears in the Schlieren image. The low level of shear for this case (as compared with Case 1) is the root cause for the delay in the break-down of these structures. Large (i.e. on the order of the jet diameter) turbulent coherent structures do not form until an $x / D$ of 15 to 20. Hence, the turbulent "stirring" processes that lead to enhanced mixing are not prevalent until the latter stages of the LES domain.

The averaged hybrid RAS/LES argon mass fraction profiles are compared with measurements in Fig. 15. Overall, the level of turbulent mixing between the argon and air jets was under-predicted. These results are comparable with those obtained using the RAS approach (see Fig. 9), though the hybrid RAS/LES shows a slightly reduced mixing level at the last experimental station. The smooth asymptotic transition of the argon profile towards zero at the edge of the mixing layer is again evident in the hybrid RAS/LES results. The reduced spreading rate of the shear layer is also seen in the Pitot pressure profile comparisons displayed in Fig. 16. The peak shear layer deficit is consistently over-predicted at each station. Future work will examine the influence of providing realistic resolved turbulent content exiting the coaxial nozzles. This may be particularly important for the present application because the boundary layer thickness of the

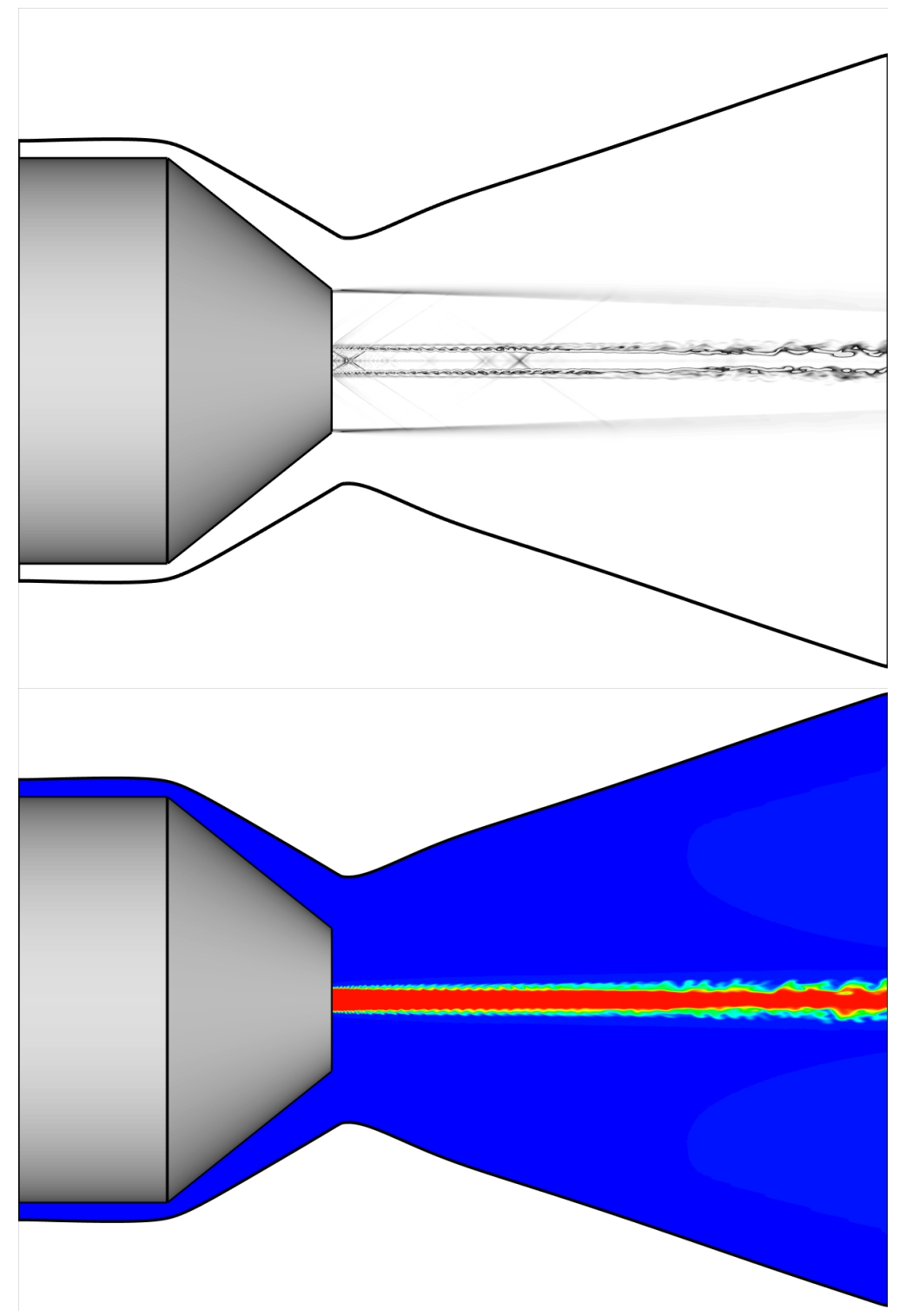

Figure 14: Instantaneous Schlieren and argon mass fraction contours (hybrid RAS/LES)

approach coflow is an order of magnitude thicker than the blunt base region which is currently triggering 
the unsteadiness. The statistics gathered after 10,000 cycles show practically identical results as the data averaged over 20,000 cycles, suggesting that the statistics are converged for these first-order correlations. The maximum azimuthal velocity (averaged over 20,000 cycles) was $0.6 \mathrm{~m} / \mathrm{s}$.

\section{Second-Order Correlations}

The primary second-order correlations that are modeled in the RAS equation set are the Reynolds stress tensor and the Reynolds heat and mass flux vectors. The Reynolds stress tensor is typically modeled by the Boussinesq approximation:

$$
\overline{\rho u_{i}^{\prime \prime} u_{j}^{\prime \prime}}=\frac{2}{3} \delta_{i j}\left(\rho k+\mu_{t} \frac{\partial u_{k}}{\partial x_{k}}\right)-\mu_{t}\left(\frac{\partial u_{i}}{\partial x_{j}}+\frac{\partial u_{j}}{\partial x_{i}}\right)
$$

and the Reynolds flux vectors commonly utilize gradient diffusion models, i.e.

$$
\overline{\rho h^{\prime \prime} u_{j}^{\prime \prime}}=-\frac{\mu_{t}}{P r_{t}} \frac{\partial h}{\partial x_{j}}
$$

and

$$
\overline{\rho Y_{m}^{\prime \prime} u_{j}^{\prime \prime}}=-\frac{\mu_{t}}{S c_{t}} \frac{\partial Y_{m}}{\partial x_{j}}
$$

The accuracy of the modeling employed for these terms is critical for the success of RAS approaches. Measured data for each of the above correlations is scarce, so it is often difficult to directly assess the accuracy of the RAS closures. One of the primary goals of this effort was to use the hybrid RAS/LES simulations to assess the performance of the RAS modeling. Ideally, one would hope for a close match between the hybrid RAS/LES results and available measurements, but the level of agreement obtained in this effort was not entirely satisfying. Nevertheless, some insight into the RAS modeling can be gleaned by comparing the modeled terms with values extracted from the resolved LES field.

The rms of the streamwise velocity fluctuation is considered first. This quantity was the only secondorder correlation that was measured, and the measurement was only taken for Case 1. These measurements were made using the RELIEF ${ }^{12}$ oxygen flow-tagging technique. Figure 17 compares the measured values with the values computed from the RAS model (Eq. 9, $S c_{t}=0.5$ ) as well as the ensemble-averages extracted from the hybrid RAS/LES. The modeled RAS results agree remarkably well with the measurements in the early stages of the shear layer development, but the width of the profile is under-predicted at stations further downstream. The hybrid RAS/LES results over-predict the rms values in the shear layer at the first station, but the profiles at the stations further downstream compare favorably with the measurements. One particular noteworthy feature is the build-up of turbulent fluctuations in the core flow near the axis that is evident in the hybrid RAS/LES results. This feature is absent from the RAS result because the mean flow gradients are relatively small outside of the shear layer. Mean velocity gradients are the sole source of turbulence production for most RAS models. The enforcement of a RAS behavior for $r / D>2$ in the hybrid RAS/LES simulations prevented the formation of unsteady turbulence structures in the outer jet/ambient air shear layer; limiting the build-up of turbulence in the core of the outer jet. Hence the core flow values in the outer jet, while larger than that predicted by pure RAS, is under-predicted.

The rms radial and azimuthal velocity fluctuations are compared in Figs. 18 and 19. Linear eddy viscosity models based on the Boussinesq approximation tend to produce nearly isotropic velocity variances (normal stresses) when applied to strain dominated flows. The degree of anisotropy is governed by the magnitude of the velocity gradients (other than those contributing to the strain rate) relative to $2 / 3 \rho k$ term (see Eq. 9). Hence, it is not surprising that the RAS model returned a nearly isotropic set of normal stress tensors for this turbulent shear flow. The hybrid RAS/LES data, on the other hand, showed a non-negligible level of anisotropy in the velocity variances. In particular, the streamwise velocity variance was substantially larger than the cross-flow variances. This result is typical for shear dominated flows.

The Reynolds shear stress $\left(\widetilde{u^{\prime \prime} v^{\prime \prime}}\right)$ and Reynolds mass flux $Y_{H e}^{\prime \prime} v^{\prime \prime}$ terms are compared in Figs. 20 and 21. The accuracy of the RAS equations, to a large extent, is driven by how well these terms are modeled. Since the $S c_{t}=1.0$ RAS results compared favorably to the measured mean flow properties, correlations from this simulation were also added to the figures. The shear stress extracted from the hybrid RAS/LES data is larger than those produced by the RAS models at the first axial two stations. The situation is reversed at the last 

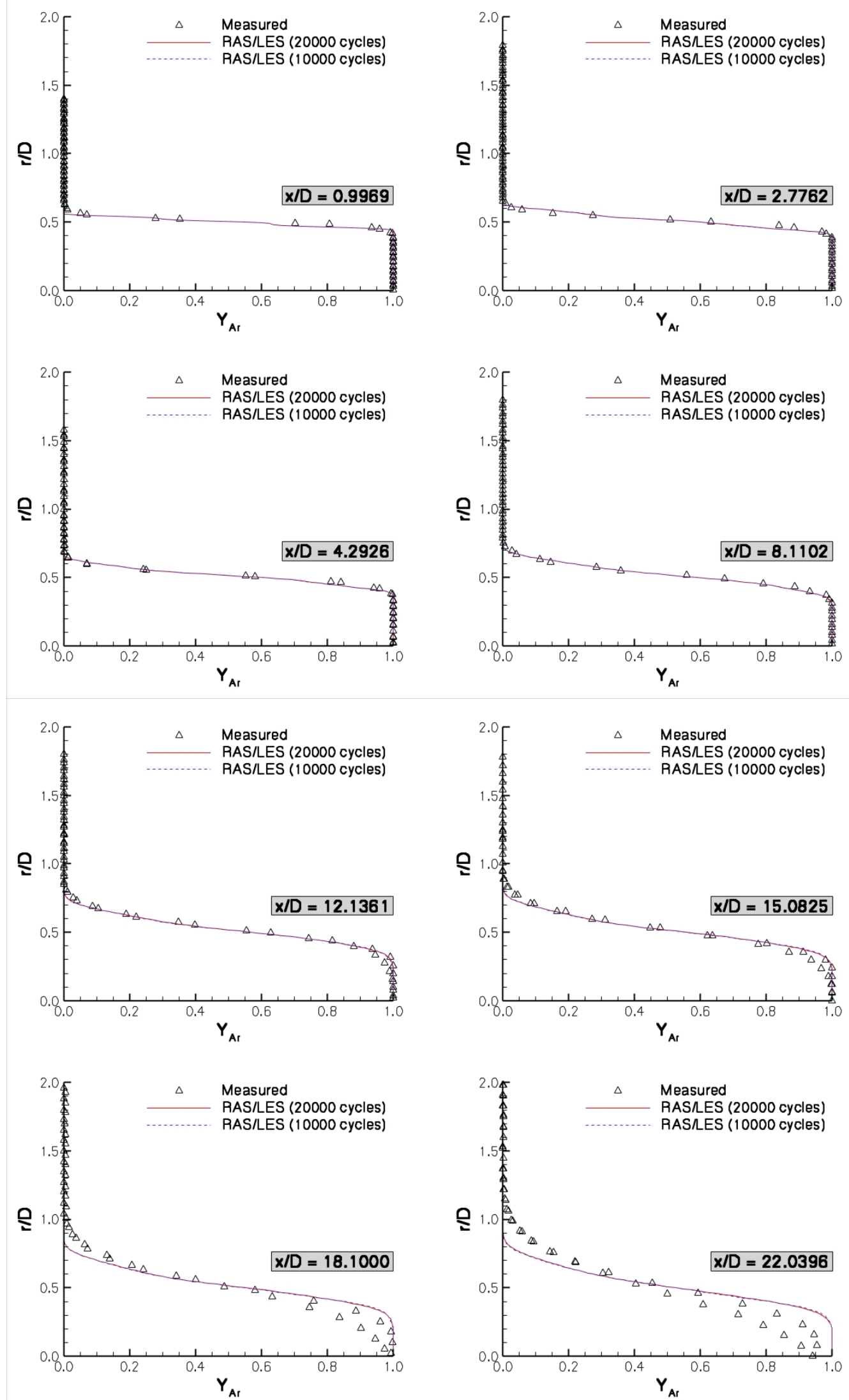

Figure 15: Comparison of argon mass fraction (averaged hybrid RAS/LES predictions) with measured values 

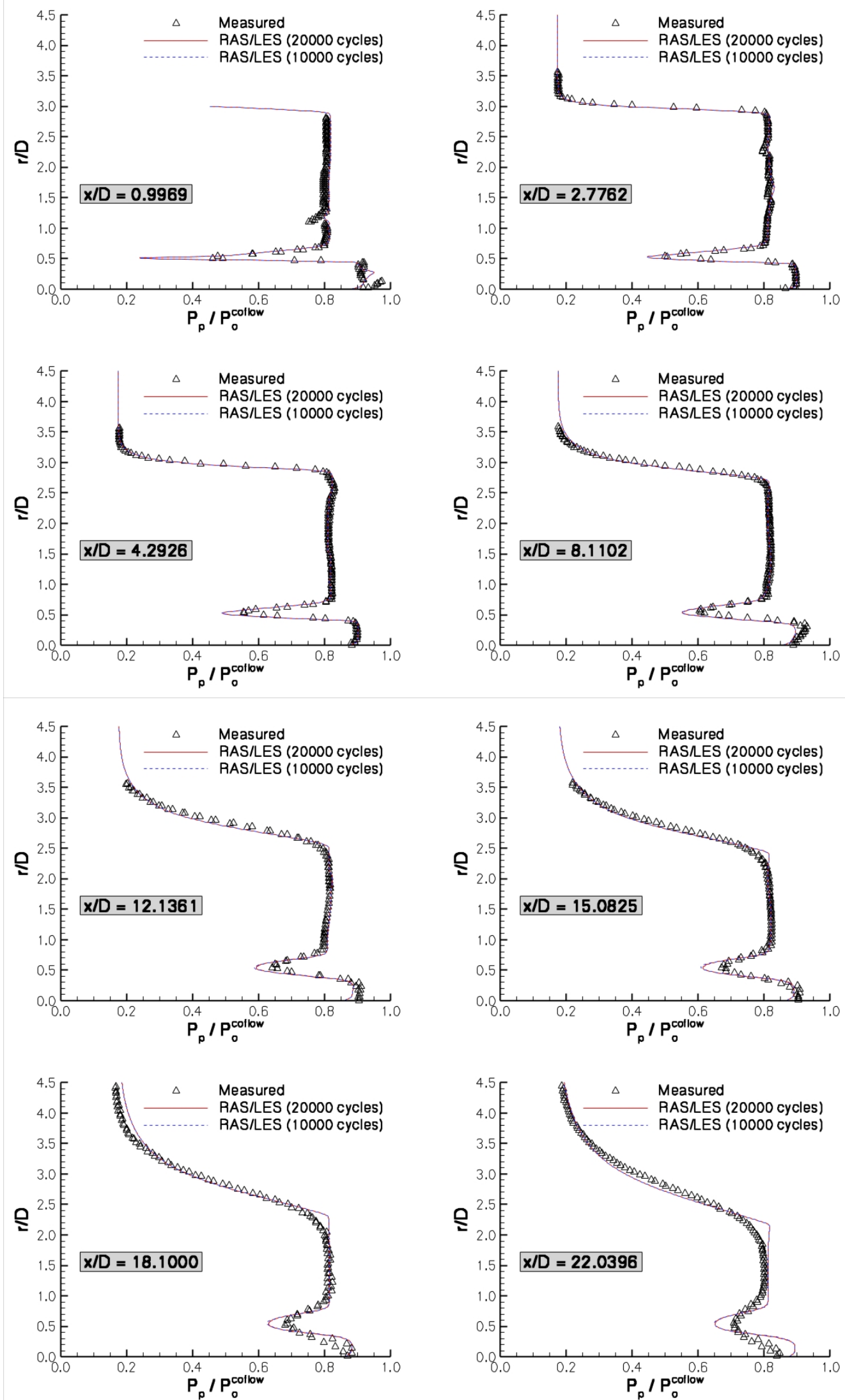

Figure 16: Comparison of Case 2 Pitot pressure (averaged hybrid RAS/LES predictions) with measured values 
two stations. This result is consistent with the fact that the hybrid RAS/LES results predicted a more rapid mixing rate than the RAS approaches. A similar behavior is evident in the Reynolds mass flux vector. The hybrid RAS/LES data showed the most rapid mixing, the $S c_{t}=1.0$ RAS yielded the slowest mixing rate, and the $S c_{t}=0.5$ RAS result was somewhere inbetween. Hence at the early axial stations, the turbulent mixing term should be largest for the hybrid RAS/LES and smallest for the $S c_{t}=1.0$ RAS. Eventually, the condition with the most efficient mixing will show a decayed mixing rate since a large fraction of the mixing process has completed. Thus at some station downstream, the simulation with the slower mixing rate should exceed that of the more rapid mixing rate. This is precisely the situation that occurs between the second and third streamwise stations shown in Fig. 21.

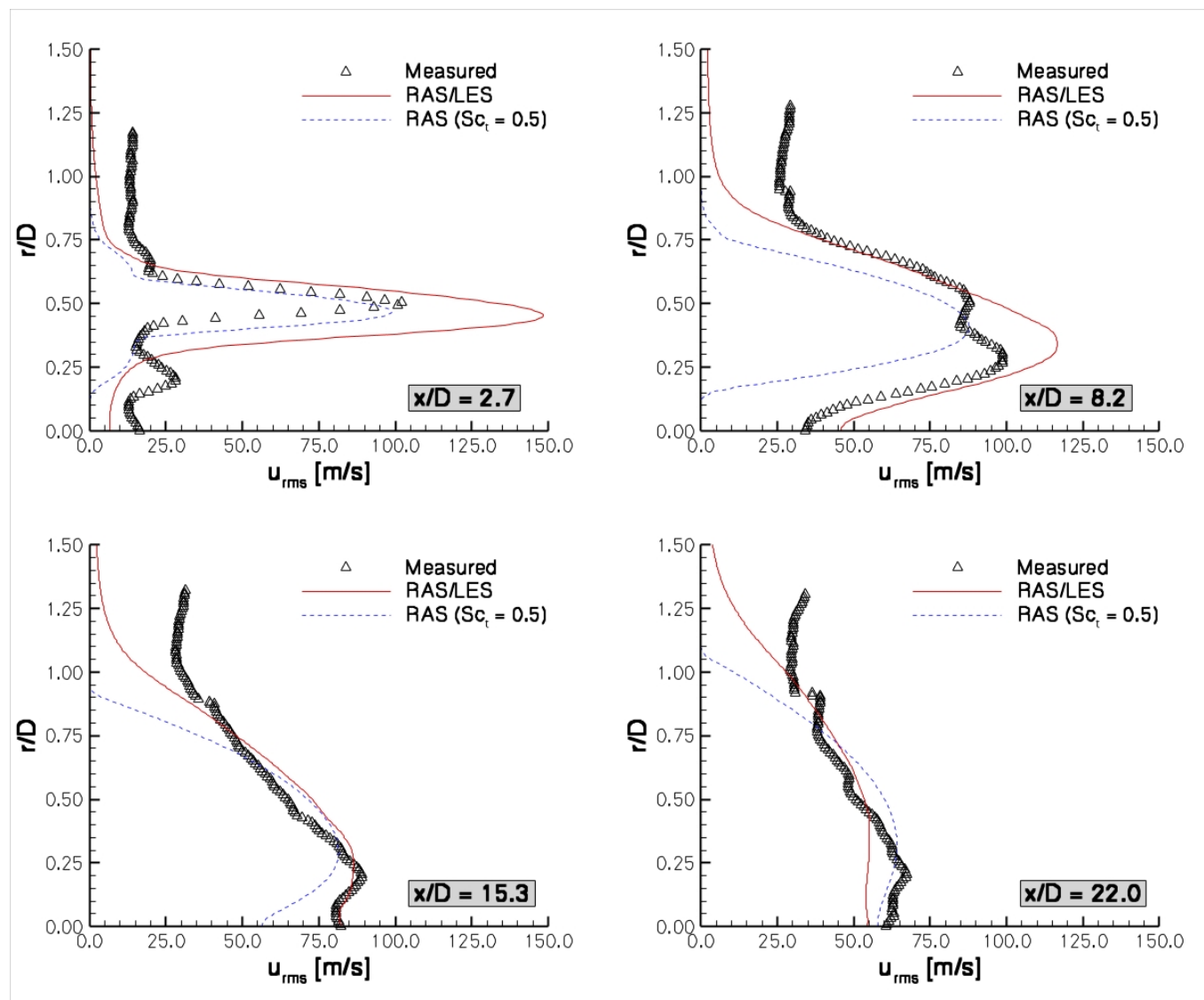

Figure 17: Comparison of Case 1 streamwise velocity fluctuation $\mathrm{rms}$ with measured values 

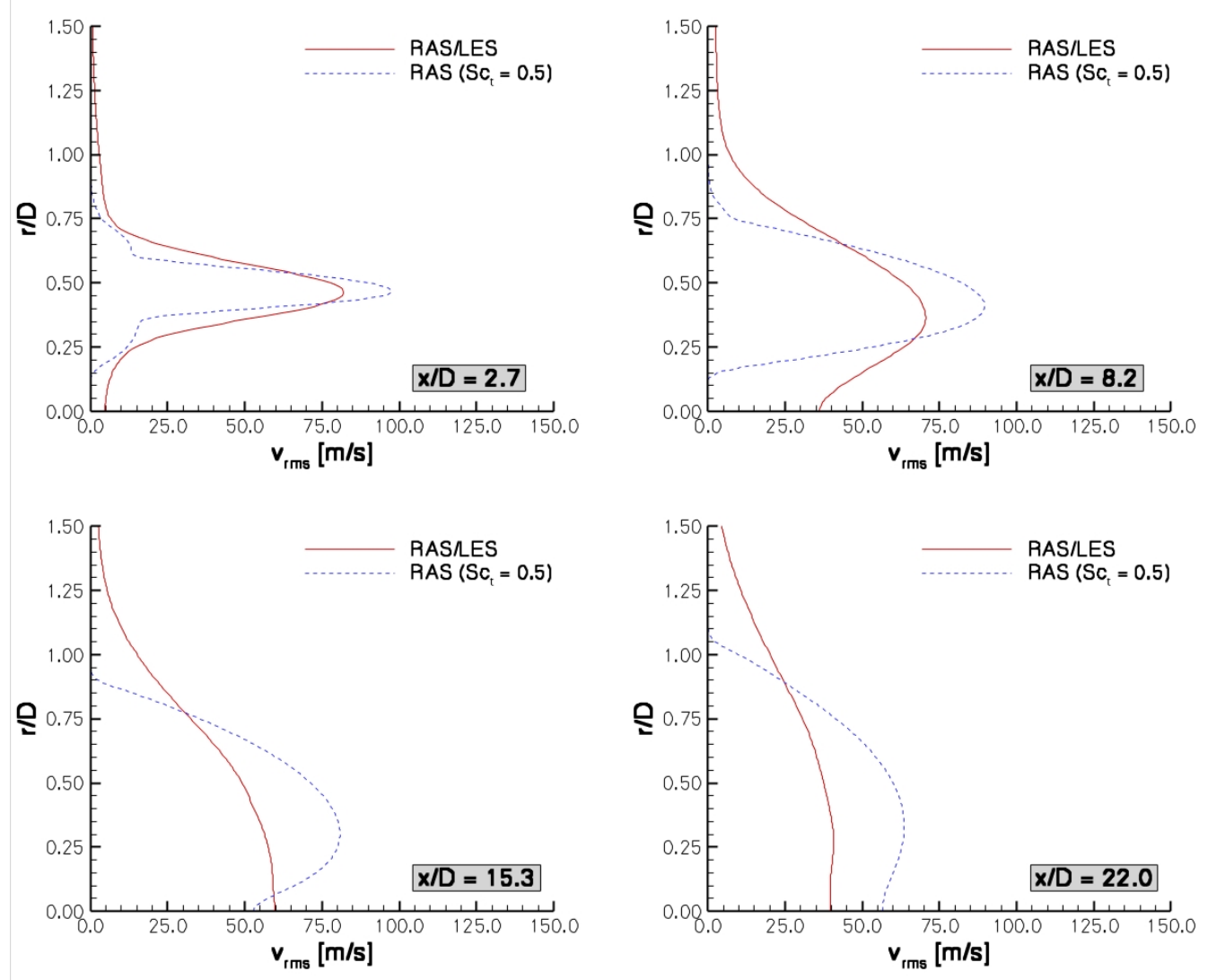

Figure 18: Comparison of Case 1 radial velocity fluctuation $\mathrm{rms}$
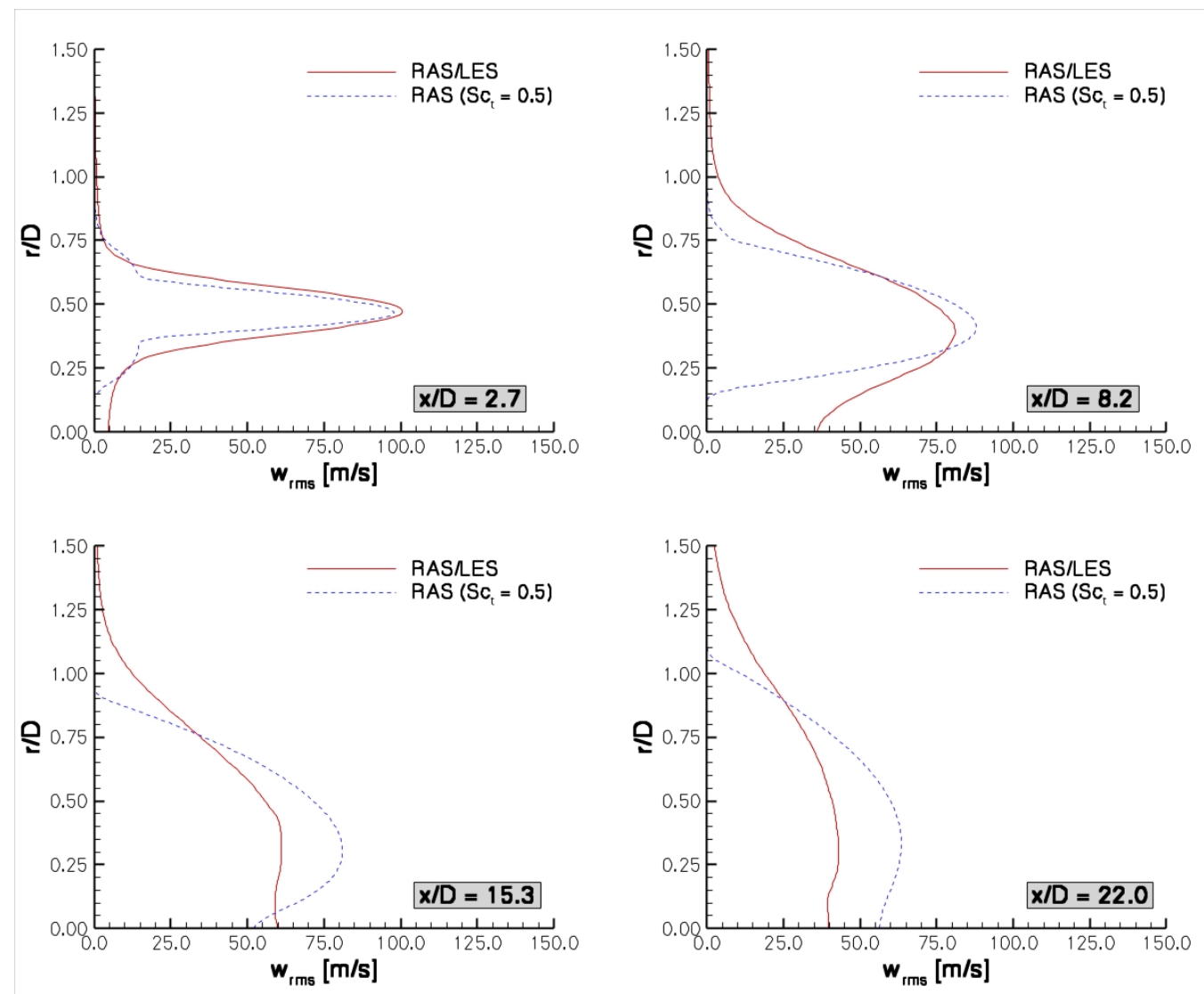

Figure 19: Comparison of Case 1 azimuthal velocity fluctuation $r m s$ 

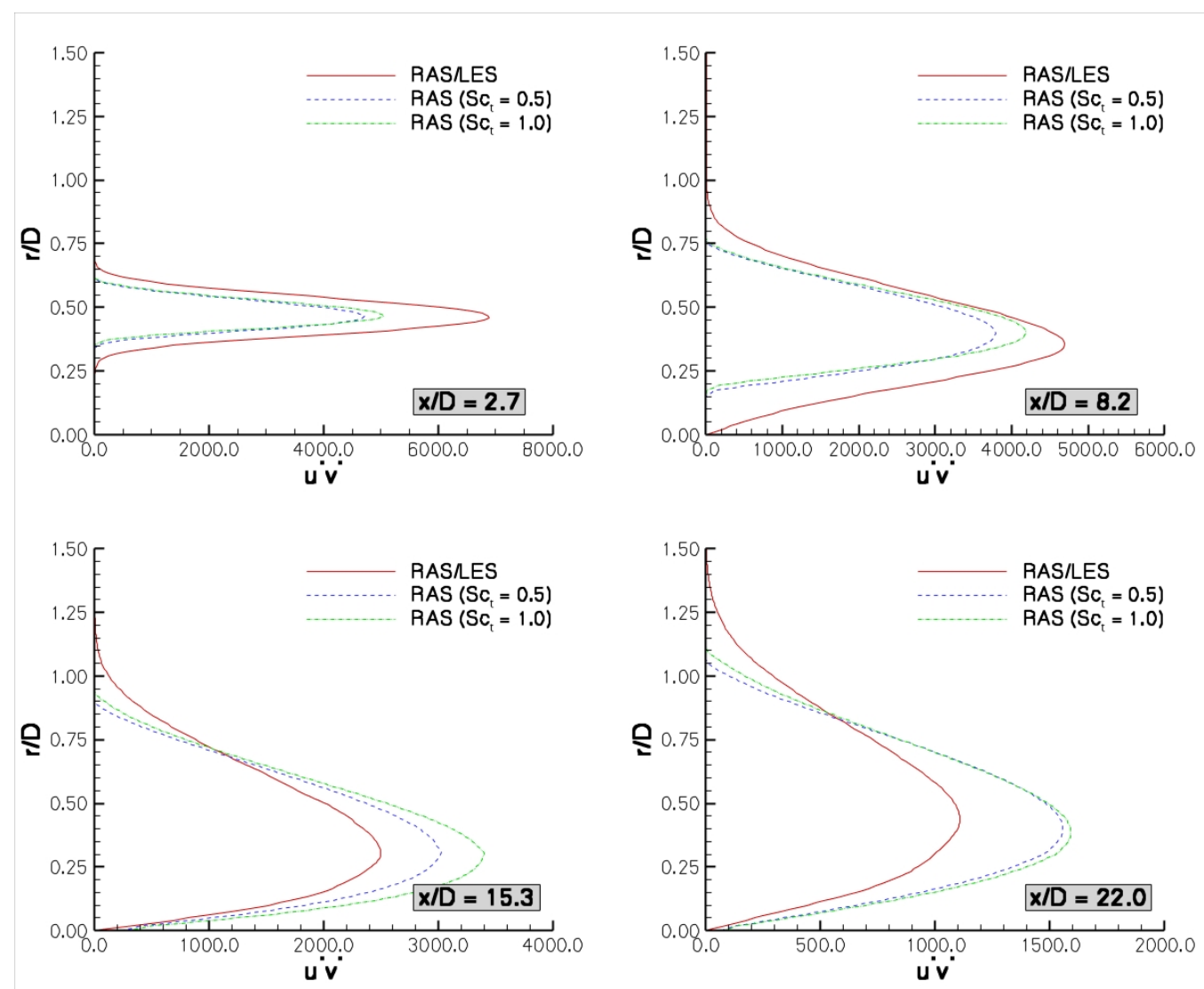

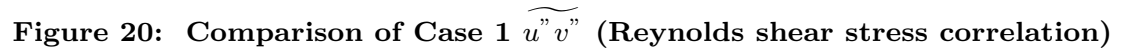
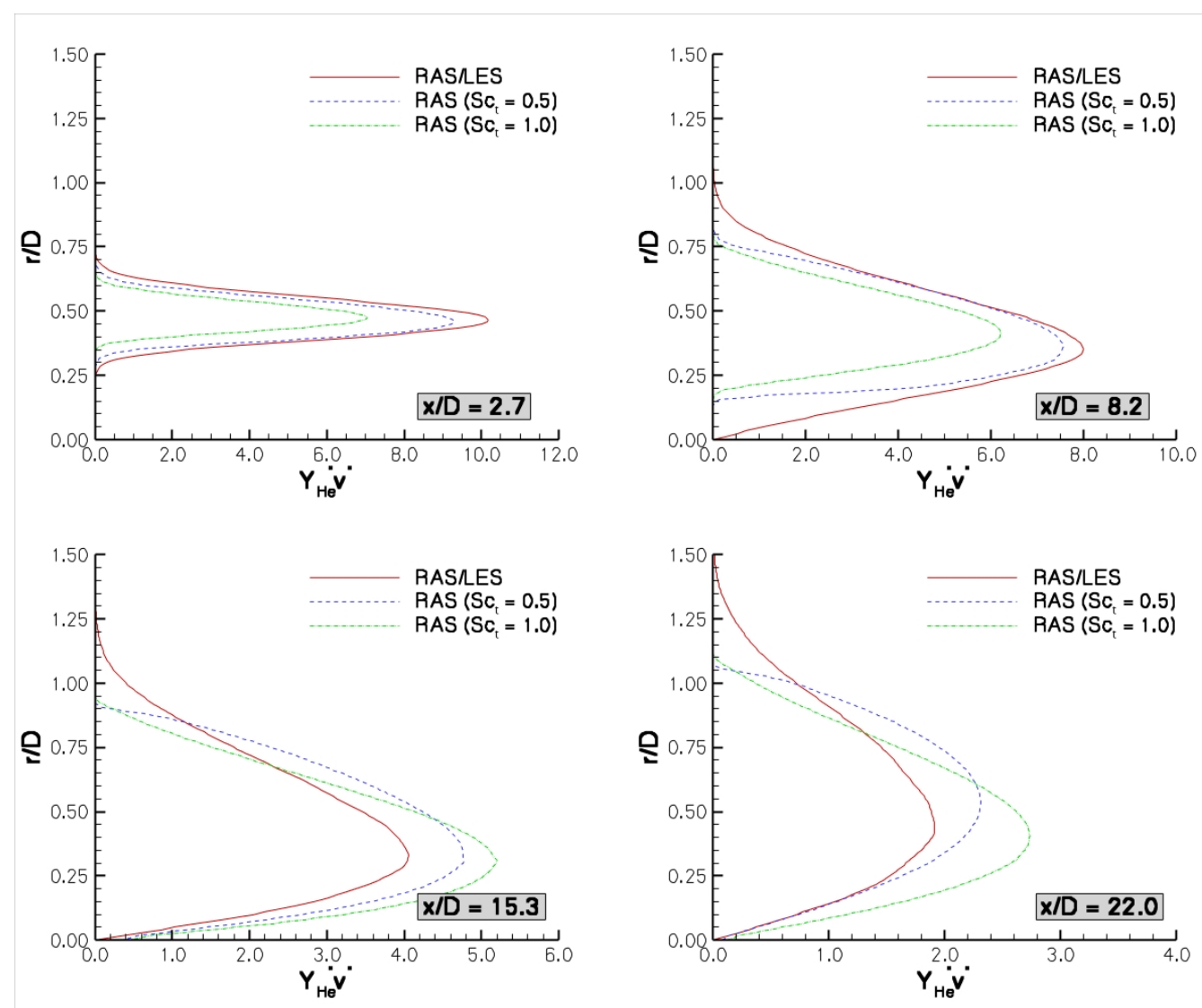

Figure 21: Comparison of $\widetilde{Y_{H e}} v^{\prime \prime}$ (Reynolds mass flux correlation) 


\section{Summary}

Reynolds-averaged and hybrid Reynolds-averaged/large-eddy simulations have been performed to model a supersonic coaxial jet flow experiment. The experiment consists of an outer jet of air, and an inner jet that was either a $\mathrm{He}-\mathrm{O}_{2}$ mixture or pure argon. Both jets exhausted into an ambient environment. The Mach 1.8 nozzle flows exiting the test apparatus were nearly pressure-matched for both injectant conditions. However, the level of shear and the compressibility of the mixing layer varied depending on the injectants involved. The helium condition resulted in a highly compressible mixing layer with a convective Mach number of 0.7 . The argon condition, on the other hand, was nearly velocity-matched and produced a mixing layer with a convective Mach number of 0.16. A comprehensive set of measurements were taken which include: Pitot pressure, mean and rms velocities, and gas sampling. The model geometry, flow conditions, and measurement uncertainties were all well documented, resulting in a package that is well suited for model validation efforts.

The goal of the computational effort was to assess the state-of-the-art for both RAS and hybrid RAS/LES approaches as applied to compressible turbulent flows. The Reynolds-averaged simulation for the helium cases displayed a strong sensitivity to choice of turbulent Schmidt number. A value of 1.0 was found to be an optimal choice for this flow condition. A lower turbulent Schmidt number, on the order of 0.5, provided the best match with measurements for the argon case, however. The uncertainty involved with appropriate choices for RAS modeling parameters highlights the difficulty with using these approaches for predictive simulations. In principal, LES or hybrid RAS/LES methods have the potential to reduce this uncertainty by resolving a substantial fraction of the turbulent flowfield. The hybrid simulations performed in this effort, however, were no more predictive than the baseline Reynolds-averaged predictions. The explanation provided for the discrepancy between the hybrid RAS/LES results and the measurements centered around issues related to how the Reynolds-averaged state transitions to a state with resolved turbulent content. The addition of resolved turbulent content to the inflow conditions was suggested to address this concern. Finally, comparisons made between resolved second-order turbulence statistics and their modeled Reynolds-averaged counterparts were discussed. Refined hybrid simulations are required, however, to improve the accuracy of the hybrid RAS/LES results before any solid conclusions can be drawn concerning the RAS modeling.

\section{Acknowledgements}

This effort was performed by the Hypersonic Airbreathing Propulsion Branch at the NASA Langley Research Center. The research was supported by the Hypersonics Project of the NASA Fundamental Aerodynamics Program.

\section{References}

\footnotetext{
${ }^{1}$ Baurle, R. A. and Eklund, D. R., "Analysis of Dual-Mode Hydrocarbon Scramjet Operation at Mach 4-6.5," Journal of Propulsion and Power, Vol. 18, No. 5, Sept.-Oct. 2002, pp. 990-1002.

${ }^{2}$ Eklund, D. R., Baurle, R. A., and Gruber, M. R., "Computational Study of a Supersonic Combustor Fueled by an Aerodynamic Ramp Injector," AIAA Paper 2001-0379, Jan. 2001.

${ }^{3}$ Rodriguez, C. G. and Cutler, A. D., "Computational Simulations of a Supersonic-Combustion Benchmark Experiment," AIAA Paper 2004-4424, July 2004.

${ }^{4}$ Spalart, P. R., Jou, W.-H., Strelets, M., and Allmaras, S. R., "Comments on the Feasibility of LES for Wings, and on a Hybrid RANS/LES Approach," 1st AFOSR International Conference on DNS/LES (invited), Aug. 1997.

${ }^{5}$ Speziale, C. G., "Turbulence Modeling for Time Dependent RANS and VLES: A Review," AIAA Journal, Vol. 36, No. 2, 1998, pp. 173-184.

${ }^{6}$ Baurle, R. A., Tam, C.-J., Edwards, J. R., and Hassan, H. A., "Hybrid Simulation Approach for Cavity Flows: Blending, Algorithm, and Boundary Treatment Issues," AIAA Journal, Vol. 41, No. 8, August 2003, pp. 1463-1480.

${ }^{7}$ Menter, F. R. and Kuntz, M., "Adaptation of Eddy-Viscosity Turbulence Models to Unsteady Separated Flow Behind Vehicles," Symposium on "The Aerodynamics of Heavy Vehicles: Trucks, Buses and Trains., edited by R. McCallen, F. Browand, and J. Ross, Springer Berlin / Heidelberg (2004), Monterey, Ca, 2002.

${ }^{8}$ Spalart, P. R., Deck, S., Shur, M. L., Squires, K. D., Strelets, M., Travin, A., "A New Version of Detached-Eddy Simulation, Resistant to Ambiguous Grid Densities," Theoretical Computational Fluid Dynamics, Vol. 20, No. 3, 2006, pp. 181195.

${ }^{9}$ Girimaji, S. S., "Partially-Averaged Navier-Stokes Model for Turbulence: A Reynolds-Averaged Navier-Stokes to Direct Numerical Simulation Bridging Method," Journal of Applied Mechanics, Vol. 73, No. 3, 2006, pp. 413-421.
} 
${ }^{10}$ Cutler, A. D., Diskin, G. S., Drummond, J. P., and White, J. A., "Supersonic Coaxial Jet Experiment for Computational Fluid Dynamics Code Validation," AIAA Journal, Vol. 44, No. 3, March 2006, pp. 585-592.

${ }^{11}$ Clifton, C. W. and Cutler, A. D., "A Supersonic Argon/Air Coaxial Jet Experiment for Computational Fluid Dynamics Code Validation," NASA Contractor Report 214866, April 2007.

${ }^{12}$ Miles, R. B., Grinstead, J., Kohl, R. Hl, and Diskin, G. S., "The RELIEF Flow Tagging Technique and its Application in Engine Testing Facilities and for Helium-Air Mixing Studies," Measurement Science Technologies, Vol. 11, 2000, pp. 1272-1281.

${ }^{13}$ White, J. A. and Morisson, J. H., "Pseudo-Temporal Multi-Grid Relaxation Scheme for Solving the Parabolized NavierStokes Equations," AIAA Paper 99-3360, June 1999.

${ }^{14}$ VULCAN, "http://vulcan-cfd.larc.nasa.gov/," Jan. 2007.

${ }^{15}$ Edwards, J. R., "A Low Diffusion Flux-Splitting Scheme for Navier-Stokes Calculations," Computers 83 Fluids, Vol. 26, No. 6, 1997, pp. 635-659.

${ }^{16}$ van Leer, B., "Towards the Ultimate Conservation Difference Scheme. II. Monotinicity and Conservation Combined in a Second Order Scheme," Journal of Computational Physics, Vol. 14, 1974, pp. 361-370.

${ }^{17}$ Wilcox, D. C., Turbulence Modeling for CFD, DCW Industries, Inc., 2nd ed., 1998.

${ }^{18}$ Wilcox, D. C., "Wall Matching, a Rational Alternative to Wall Functions," AIAA Paper 89-0611, Jan. 1989.

${ }^{19}$ Suresh, A. and Huynh, H. T., "Numerical Experiments on a New Class of Nonoscillatory Schemes," AIAA Paper 92-0421, Jan. 1992.

${ }^{20}$ Yoshizawa, A. and Horiuti, K., "A Statistically-Derived Subgrid Scale Kinetic Energy Model for Large-Eddy Simulation of Turbulent Flows," Journal of the Physical Society of Japan, Vol. 54, 1985, pp. 2834-2839.

${ }^{21}$ Strelets, M., "Detached Eddy Simulation of Massively Separated Flows," AIAA Paper 2001-0879, Jan. 2001.

${ }^{22}$ Nikitin, N. V., Nicoud, F., Wasistho, B. Squires, K. D., and Spalart, P. R., "An Approach to Wall Modeling in Large-Eddy Simulations," Physics of Fluids, Vol. 12, July 2000, pp. 1629-1632.

${ }^{23}$ Choi, J., Edwards, J. R., and Baurle, R. A., "Compressible Boundary Layer Predictions at High Reynolds Number Using Hybrid LES/RANS Methods," AIAA Paper 2008-4175, June 2008.

${ }^{24}$ Ferziger, J. H., "Large Eddy Simulation," March 1994, ICASE/LaRC Short Course on Turbulent Flow Modeling and Prediction.

${ }^{25}$ TECPLOT, "http://www.tecplot.com/," Jan. 2009.

${ }^{26}$ Xiao, X., Edwards, J. R., Hassan, H. A., and Baurle, R. A., "Inflow Boundary Conditions for Hybrid Large Eddy / Reynolds Averaged Navier-Stokes Simulations," AIAA Journal, Vol. 41, No. 8, August 2003, pp. 1481-1489.

${ }^{27}$ Sagaut, P., Garnier, E., Tromeur, E., Larchêveque, L., and Labourasse, E., "Turbulent Inflow Conditions for Large Eddy Simulation of Compressible Wall-Boundary Flows," AIAA Journal, Vol. 42, No. 3, 2004, pp. 469-477.

${ }^{28} \mathrm{Xu}$, S. and Martin, M. P., "Assessment of Inflow Boundary Conditions for Compressible Turbulent Boundary Layers," Physics of Fluids, Vol. 16, No. 7, 2004, pp. 2623-2639. 